\title{
Foxf2 plays a dual role during transforming growth factor beta-induced epithelial to mesenchymal transition by promoting apoptosis yet enabling cell junction dissolution and migration
}

Nathalie Meyer-Schaller ${ }^{1,2+}$, Chantal Heck ${ }^{1,3+}$, Stefanie Tiede ${ }^{1}$, Mahmut Yilmaz ${ }^{1,4}$ and Gerhard Christofori ${ }^{1 *}$ (D)

\begin{abstract}
Background: The most life-threatening step during malignant tumor progression is reached when cancer cells leave the primary tumor mass and seed metastasis in distant organs. To infiltrate the surrounding tissue and disseminate throughout the body, single motile tumor cells leave the tumor mass by breaking down cell-cell contacts in a process called epithelial to mesenchymal transition (EMT). An EMT is a complex molecular and cellular program enabling epithelial cells to abandon their differentiated phenotype, including cell-cell adhesion and cell polarity, and to acquire mesenchymal features and invasive properties.

Methods: We employed gene expression profiling and functional experiments to study transcriptional control of transforming growth factor (TGF) $\beta$-induced EMT in normal murine mammary gland epithelial (NMuMG) cells.

Results: We identified that expression of the transcription factor forkhead box protein F2 (Foxf2) is upregulated during the EMT process. Although it is not required to gain mesenchymal markers, Foxf2 is essential for the disruption of cell junctions and the downregulation of epithelial markers in NMuMG cells treated with TGF $\beta$. Foxf2 is critical for the downregulation of E-cadherin by promoting the expression of the transcriptional repressors of E-cadherin, Zeb1 and Zeb2, while repressing expression of the epithelial maintenance factor Id2 and miRNA 200 family members. Moreover, Foxf2 is required for TGF $\beta$-mediated apoptosis during EMT by the transcriptional activation of the proapoptotic BH3only protein Noxa and by the negative regulation of epidermal growth factor receptor (EGFR)-mediated survival signaling through direct repression of its ligands betacellulin and amphiregulin. The dual function of Foxf2 during EMT is underscored by the finding that high Foxf2 expression correlates with good prognosis in patients with early noninvasive stages of breast cancer, but with poor prognosis in advanced breast cancer.
\end{abstract}

Conclusions: Our data identify the transcription factor Foxf2 as one of the important regulators of EMT, displaying a dual function in promoting tumor cell apoptosis as well as tumor cell migration.

Keywords: Apoptosis, Breast cancer, Cell migration, E-cadherin, EGFR, EMT, Foxf2, Noxa

\footnotetext{
* Correspondence: gerhard.christofori@unibas.ch

${ }^{\dagger}$ Nathalie Meyer-Schaller and Chantal Heck contributed equally to this work.

'Department of Biomedicine, University of Basel, Mattenstrasse 28, 4058

Basel, Switzerland

Full list of author information is available at the end of the article
}

(c) The Author(s). 2018 Open Access This article is distributed under the terms of the Creative Commons Attribution 4.0 International License (http://creativecommons.org/licenses/by/4.0/), which permits unrestricted use, distribution, and reproduction in any medium, provided you give appropriate credit to the original author(s) and the source, provide a link to the Creative Commons license, and indicate if changes were made. The Creative Commons Public Domain Dedication waiver (http://creativecommons.org/publicdomain/zero/1.0/) applies to the data made available in this article, unless otherwise stated. 


\section{Background}

The process of epithelial to mesenchymal transition (EMT) describes a complex molecular and cellular program by which epithelial cells abandon their differentiated features and acquire mesenchymal characteristics, including motility, invasiveness, and increased resistance to apoptosis [1-5]. EMT has been implicated in several physiological as well as pathological processes. While it is a critical mechanism for embryonic development, EMT is re-engaged in adults during wound healing, tissue regeneration, organ fibrosis, and cancer progression and metastasis [2]. Recent studies implicate that primary tumors displaying an EMT-like gene expression profile are more likely to be associated with distant metastasis formation and a worse prognosis for overall survival [6-8]. In contradiction to these findings are the observations that distant metastases frequently exhibit an epithelial phenotype highly similar to the primary tumor $[9,10]$. Explaining this observation, it has been shown that disseminated mesenchymal cancer cells undergo the reverse process (mesenchymal to epithelial transition (MET)) after metastatic spread and colonization and revert to a differentiated, epithelial cell state enabling them to establish in the distant location [11-13]. However, the contribution of EMT to the metastatic process is debated. Recent lineage tracing experiments have suggested that EMT is required for the development of drug resistance but not for metastasis $[14,15]$. However, these reports have been met with great skepticism and data questioning these results [16-18]. In summary, overwhelming evidence supports the conclusion that EMT and its reverse process MET are pivotal regulators of cell plasticity in malignant tumor progression and play important roles in drug resistance, relapse, and metastatic progression [19].

Recently, a number of transcription factors have been identified that play critical roles in the initiation and execution of an EMT and in the metastatic process, including Snai1 (Snail), Snai2 (Slug), Zeb1 (SEF1), Zeb2 (Sip1), E47, Twist, goosecoid, Foxc2, Dlx2, RBPjк, Yap/Taz, Sox4 and 9, Klf4, and NFKB [3, 19-22]. We have previously established a list of genes that change in their expression during the consecutive morphological states of transforming growth factor (TGF) $\beta$-induced EMT in normal murine mammary gland (NMuMG) epithelial cells $[20,23]$. This analysis identified forkhead box protein F2 (Foxf2) as a transcription factor that is upregulated in its expression during EMT in NMuMG cells and in several other experimental EMT systems. The family of Forkhead box (Fox) genes are defined by a conserved DNA binding domain of a winged helix structure acting as transcription factors, which have been found to serve as key regulators in embryogenesis, signal transduction, maintenance of differentiated cell states, and tumorigenesis [24]. There are three families of Fox genes, Foxc, Foxf, and Foxl1, that form paralogous clusters in the genome and that are extensively expressed in mesodermal tissue $[25,26]$. One of the best characterized members of this family is Foxc2, which has been implicated in the regulation of EMT by interacting with Smad proteins and to be a key player in metastasis [27, 28]. Moreover, Foxc1 and Foxc2 are highly expressed in the claudin-low metaplastic breast cancer subtype, which is associated with EMT and cancer stemness [29]. Furthermore, the overexpression of Foxm1 in pancreatic cancer cells leads to the acquisition of an EMT phenotype via upregulation of Zeb1 and Zeb2 as well as stem cell-like characteristics [30].

Foxf2 (also known as Freac-2 or Fkhl6) is a widely expressed protein in various mesenchymal tissues and was first identified as a transcriptional activator containing a forkhead domain for nuclear localization and two independent C-terminal activation domains [31]. Foxf2 interacts with TBP and TFIIB, two components of the general transcriptional activator complex binding a specific DNA motif $[32,33]$. Expression of several Fox family genes, including Foxf1 and Foxf2, is specifically regulated by sonic hedgehog (Shh) signaling in a crosstalk with Notch, epidermal growth factor (EGF)/fibroblast growth factor (FGF), and TGF $\beta$ signaling $[34,35]$. Indeed, TGF $\beta$-induced EMT is one of the mechanisms strongly involved in regulating fusion of the palatal cleft, and Foxf2 levels are high in the mesenchyme of the secondary palate and in the mesenchyme of the lung and gut $[36,37]$. Accordingly, Foxf2-deficient mice die shortly after birth due to cleft palate and abnormal tongue and gut development, indicating an essential role of Foxf2 in this EMT-associated developmental process [38, 39]. Epithelial cells of Foxf2-deficient mice show typical signs of depolarization, and the subcellular localization of adherens junctions, normally confined to lateral membranes, expands into the basal and apical membranes.

In many cancer types, the expression of Foxf2 is repressed by promoter hypermethylation or by oncogenic microRNAs (miRNAs), such as miR-301, which promotes breast cancer cell proliferation, invasion, and tumor growth $[40,41]$, indicating that Foxf2 may act as a tumor suppressor. However, other studies have reported a protumorigenic role of Foxf 2 in other cancer types (reviewed in [41]), for example by repressing intestinal stem cells and preventing adenoma formation by inhibiting Wnt signaling [42]. Furthermore, low Foxf2 expression has been reported to correlate with early-onset metastasis and poor prognosis in breast cancer patients [43], and loss of Foxf2 expression promotes an EMT and metastasis of experimental cancer $[44,45]$. These conflicting results seem to mirror a double-sided role for Foxf2 in maintaining tissue homeostasis, in regulating an EMT, and in breast cancer progression [46]. 
Here, we have employed a TGF $\beta$-induced EMT in NMuMG cells and in murine and human breast cancer cells to demonstrate a critical role of Foxf2 during an EMT by concomitantly regulating an EMT, cell survival, and apoptosis. The mechanistic insights into Foxf2 functions also support a dual role of Foxf2 in breast cancer progression and metastasis, on one hand by affecting cell junction homeostasis, and by regulating cell proliferation and survival on the other hand.

\section{Methods}

Reagents and antibodies

See Additional file 1.

\section{Cell culture and cell lines}

All reagents used for cell culture were obtained from Sigma/Fluka (Basel, Switzerland) if not otherwise mentioned. All cells were cultured at $37{ }^{\circ} \mathrm{C}$ with $5 \% \mathrm{CO}_{2}$ in Dulbecco's modified Eagle's medium (DMEM) supplemented with glutamine (2 $\mathrm{mM})$, penicillin (100 U), streptomycin $(0.2 \mathrm{mg} / \mathrm{l})$ and $10 \%$ fetal bovine serum (FBS). The subclone NMuMG/E9 (hereafter called NMuMG) is expressing E-cadherin and has previously been described [47]. MTAEcad and MCF7-shEcad have been described previously [23]. NMuMG-shSmad4 and NMuMG-shCtr were obtained from P. ten Dijke (Leiden University Medical Center, The Netherlands) [48]. Py2T breast cancer cells were established from a tumor of the MMTV-PyMT mouse model of breast cancer as previously described [49]. NMuMG cells were treated with TGF $\beta(2 \mathrm{ng} / \mathrm{ml})$ without serum deprivation, and TGF $\beta$ was replenished every 2 days. siRNA transfections with lipofectamine RNAiMAX (Invitrogen) were performed according to the manufacturer's protocol $24 \mathrm{~h}$ before treatment with TGF $\beta$.

\section{Generation of lentivirus}

A cDNA encoding Foxf2 (kindly provided by Leif Lundh, Goteborg University, Sweden) [50] was tagged $\mathrm{N}$-terminally with HA-tag and cloned into the lentiviral expression vector pLenti-CMV-Puro (kindly provided by Matthias Kaeser, Bern). Lentiviral particles were produced by transfecting HEK293T cells with the lentiviral expression vectors in combination with the packaging vector pR8.91 and the envelope encoding vector pVSV using Fugene HD (Roche). After 2 days, the virus-containing HEK293T supernatant was harvested, filtered $(0.45 \mu \mathrm{m})$, supplemented with polybrene $(8 \mathrm{ng} / \mathrm{ml})$, and used for $\operatorname{tar}$ get cell infection. Infections were performed twice a day on 2 consecutive days.

\section{Growth curves}

One day before $t_{0}, 1.6 \times 10^{4}$ NMuMG cells were seeded in triplicate into 24-well plates and transfected with the indicated siRNA. After $24 \mathrm{~h}$ the cells were treated with TGF $\beta$ and cell numbers were determined using a Neubauer counting chamber.

\section{Migration assay}

NMuMG cells $\left(2 \times 10^{4} /\right.$ well $)$ pretreated for 18 days with TGF $\beta$ were seeded in DMEM, $2 \%$ FBS, and TGF $\beta$ into the upper chamber of a cell culture insert (pore size $8 \mu \mathrm{m}$; Falcon BD, Franklin Lakes, NJ). The lower chamber was filled with DMEM, 20\% FBS, and TGF $\beta$. After $16 \mathrm{~h}$ incubation at $37{ }^{\circ} \mathrm{C}$ and $5 \% \mathrm{CO}_{2}$, the cells that had traversed the membrane were fixed in $4 \%$ paraformaldehyde/phosphate-buffered saline (PBS) (15 min at room temperature), stained with DAPI $(0.5 \mu \mathrm{g} / \mathrm{ml})$, and counted using a fluorescence microscope.

\section{Quantitative real-time polymerase chain reaction (RT-PCR)} Total RNA was prepared using Tri Reagent (Sigma-Aldrich), reverse transcribed with ImProm-II Reverse Transcriptase (Promega) and transcription levels were quantified using SYBR-green PCR Mastermix (Eurogentec) in a real-time PCR system (Step One Plus, Applied Biosystems). Human or mouse riboprotein L19 primers were used for normalization. PCR assays were performed in duplicate and the fold induction was calculated against control-treated cells using the comparative Ct method $\left(\Delta \Delta C_{t}\right)$. To quantify miRNA levels, RNA was isolated with the miRNeasy kit (Qiagen) followed by polyadenylation and reverse transcription using QuantiMir RT kit (BioCat). Primers are listed in Additional file 1.

\section{Immunoblotting and immunofluorescence staining}

See Additional file 1.

\section{Apoptosis assay}

Cells were washed twice in ice-cold PBS and suspended in $1 \times$ Annexin-V binding buffer (0.01 M HEPES, pH 7.4, $0.14 \mathrm{M} \mathrm{NaCl}, 2.5 \mathrm{mM} \mathrm{CaCl}_{2}$,) at a concentration of $1 \times 10^{6}$ cells/ml; $5 \mu \mathrm{l}$ of Cy 5 Annexin-V was added to $1 \times 10^{5}$ cells and incubated for $15 \mathrm{~min}$ on ice in the dark. Stained cells were filtered through a $40-\mu \mathrm{m}$ mesh and analyzed on a FACSCanto II using DIVA Software (Becton Dickinson). Cell debris and duplets were excluded by a combination of light scatter and forward scatter plus width.

\section{Cell cycle analysis}

Cells were incubated with $10 \mu \mathrm{M}$ BrdU for $2 \mathrm{~h}$ at $37{ }^{\circ} \mathrm{C}$ and $5 \% \mathrm{CO}_{2}$. The cells were then fixed in $70 \%$ ice-cold ethanol and lysed by incubating first with $2 \mathrm{~N} \mathrm{HCl}$ and $0.5 \%$ Triton $\mathrm{X}-100$ for $30 \mathrm{~min}$ and then in $0.1 \mathrm{M}$ $\mathrm{Na}_{2} \mathrm{~B}_{4} \mathrm{O}_{7}, \mathrm{pH} 8.5$, for $2 \mathrm{~min}$ at room temperature. Nuclei were washed with $0.5 \%$ Tween-20, $1 \%$ bovine serum albumin (BSA)/PBS and incubated with FITC-labeled anti-BrdU antibody (\#347583, Beckton Dickinson) for 
$30 \mathrm{~min}$ at room temperature. Nuclei were stained for DNA content by incubating with $5 \mu \mathrm{g} / \mathrm{ml}$ propidium iodide (PI) for a minimum of $1 \mathrm{~h}$ at room temperature. Stained cells were filtered through a $40-\mu \mathrm{m}$ mesh and analyzed on a FACSCanto II using DIVA Software (Becton Dickinson).

\section{Chromatin immunoprecipitation}

Chromatin immunoprecipitation (ChIP) experiments were performed as previously described with some modifications [51]. In brief, cells were crosslinked either with $1 \%$ formaldehyde or in combination with $2 \mathrm{mM}$ EGS (ethylene glycol bis(succinimidyl succinate); ThermoFisher, 21,565). Crosslinked chromatin was sonicated to receive an average fragment size of $500 \mathrm{bp}$. ChIP was performed with $100 \mu \mathrm{g}$ of chromatin and 2.5-5 $\mu \mathrm{g}$ HA-tag antibody per IP, and 1\% of ChIP material or input material was used for quantitative RT-PCR using specific primers covering Foxf2 binding sites in promoter regions of Btc ( -450 to -253 from TSS), of Ereg (-851 to -654 from TSS), of Areg exon2 (+1086 to 1210 from TSS), and of Noxa ( -696 to -499 from TSS). Primers covering an intergenic region were used as control, and the amplification efficiencies were normalized between the primer pairs. Enrichment of IP/input over IgG background control was calculated and the specificity measured as fold change to an unspecific intergenic region.

Transcriptome, survival, and metastasis correlation analysis See Additional file 1.

\section{Statistical analysis}

Statistical analysis and graphs were generated using the GraphPad Prism software (GraphPad Software Inc., San Diego CA). All statistical analyses were performed as indicated by paired or unpaired two-sided $t$ test.

\section{Results}

\section{Foxf2 expression is induced during EMT}

We screened for changes in gene expression by DNA oligonucleotide microarray analysis during an EMT in three independent in vitro model systems. First, MTflEcad cells have been derived from a breast tumor of MMTV-Neu transgenic mice [52] in which both E-cadherin alleles were flanked by LoxP recombination sites [53]. Genetic ablation of E-cadherin was achieved by the transient expression of Cre-recombinase (MT $\Delta \mathrm{E}$ cad) [23]. Second, EMT was induced in the human breast cancer cell line MCF7 by downregulation of E-cadherin using stable expression of shRNA [23] and, thirdly, EMT was induced in normal murine mammary epithelial (NMuMG) cells by treatment with TGF $\beta$ [54] (Additional file 1: Figure S1A). The forkhead transcription factor Foxf2 was identified as a commonly upregulated gene during EMT in all three experimental systems (Additional file 1: Figure S1B, C). To assess whether Foxf2 is a target of canonical or noncanonical TGF $\beta$ signaling, we monitored Foxf2 expression in NMuMG cells stably depleted of Smad4 expression (NMuMG-shSmad4) [48]. Foxf2 mRNA expression levels were significantly reduced in TGF $\beta$-treated NMuMG-shSmad4 cells compared with control cells, indicating that Foxf2 is regulated via canonical Smad4-dependent TGF $\beta$ signaling (Additional file 1: Figure S1D).

\section{Foxf2 is partially required for EMT}

We first assessed whether the expression of Foxf2 is able to induce an EMT by infecting NMuMG cells with lentiviral particles encoding HA-tagged human Foxf2. Although the cells expressed Foxf2 in their nuclei, the cells did not gain an EMT-like phenotype (data not shown). Conversely, to investigate whether the upregulation of Foxf2 expression is required for an EMT, we stably infected NMuMG cells with lentiviral particles expressing two different shRNAs against murine Foxf2 (shFoxf2 \#703, shFoxf2 \#704). NMuMG-shFoxf2 cells treated with TGF $\beta$ apparently changed to a mesenchymal cell morphology, comparable to TGF $\beta$-treated NMuMG-shCtrl cells. However, NMuMG-shFoxf2 cells did not completely lose their tight cell-cell contacts, a key step during an EMT (Fig. 1a). Indeed, quantitative RT-PCR (Fig. 1b, c) and immunoblotting (Fig. 1d) analysis revealed that the shRNA-mediated ablation of Foxf2 expression resulted in a sustained expression of the epithelial adherens and tight junction molecules E-cadherin and ZO-1, whereas the increased expression of the mesenchymal markers fibronectin, Ncam1, and $\mathrm{N}$-cadherin remained unaffected.

To investigate whether the shRNA-mediated depletion of Foxf2 expression affects EMT-associated changes in cell adhesion, cell junctions, and/or cytoskeletal composition, we performed immunofluorescence microscopy analysis for the cell adhesion proteins E-cadherin, N-cadherin, and Ncam1, the tight junction protein $\mathrm{ZO}-1$, the focal adhesion protein paxillin, and actin stress fibers (phalloidin). NMuMG-shFoxf2 cells did not show a classical cadherin switch when treated with TGF $\beta$. In Foxf2-ablated cells, a normal upregulation of the mesenchymal marker $\mathrm{N}$-cadherin was observed, but the expression of the epithelial markers E-cadherin and ZO-1 was partially maintained at the cell membrane, in contrast to shCtrl-expressing cells which showed a bona-fide EMT (Additional file 1: Figure S2A, B). Upregulated expression of Foxf2 during an EMT was also not required for the EMT-associated cytoskeletal reorganization of cortical actin into actin stress fibers, for the 
A

+ TGF $\beta$ (days)



$1 d$
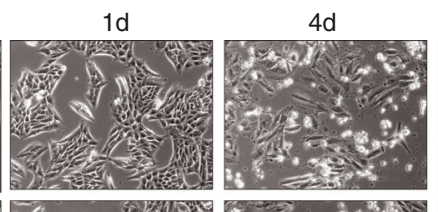

$7 d$

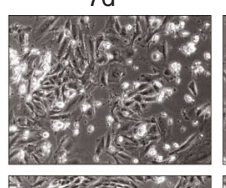

$10 d$
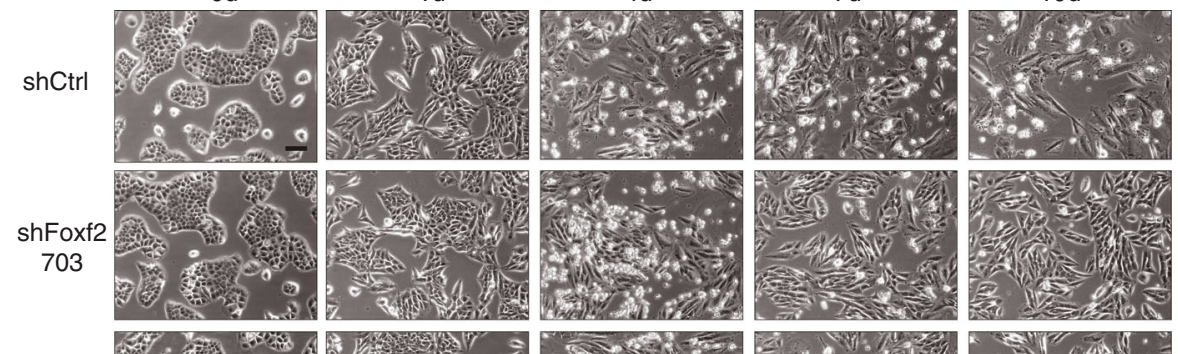

shFoxf2

704
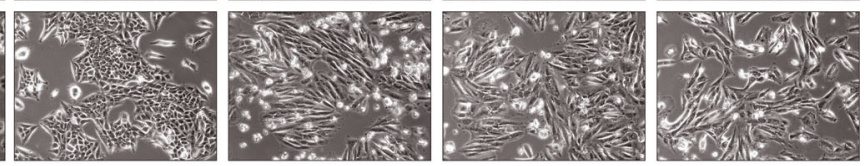

B

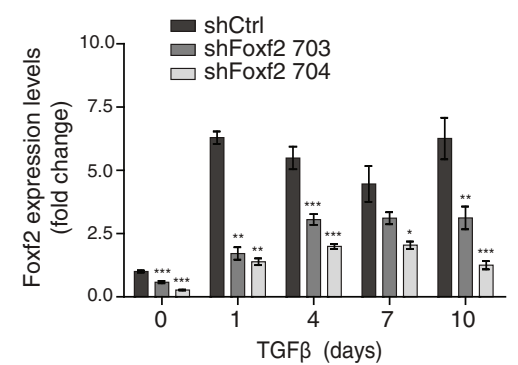

D

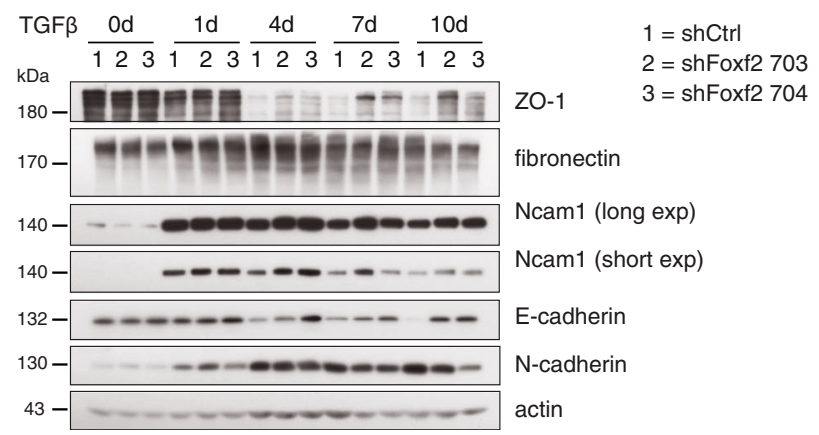

C

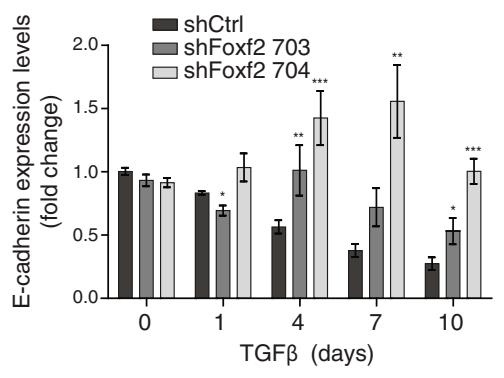

$=$ shCtrl

= shFoxf 703$$
\text { . }
$$ 
cellular migration (Fig. 2a-d). Since Foxf2 appeared essential for the loss of E-cadherin expression and the disruption of cell-cell junctions, we assessed whether the loss of Foxf2 expression affected the migratory capabilities of cells. Transwell migration assays revealed a decrease in motility for cells stably expressing shRNAs against murine Foxf2 compared with NMuMG cells expressing control shRNA (Fig. 2e).

\section{A}

20d TGF $\beta$

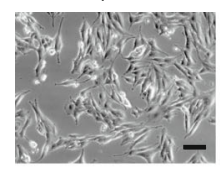

shCtrl

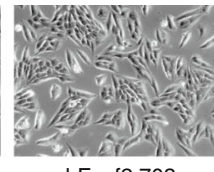

shFoxf2 703

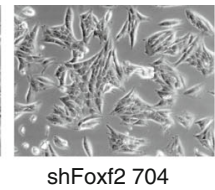

C

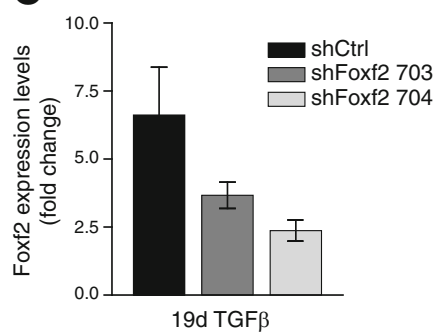

E

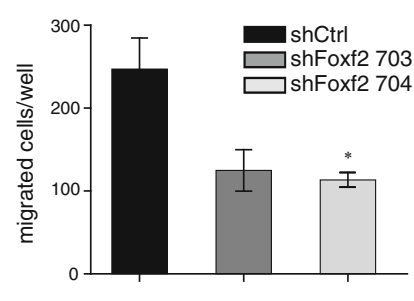

19d TGF $\beta$

G

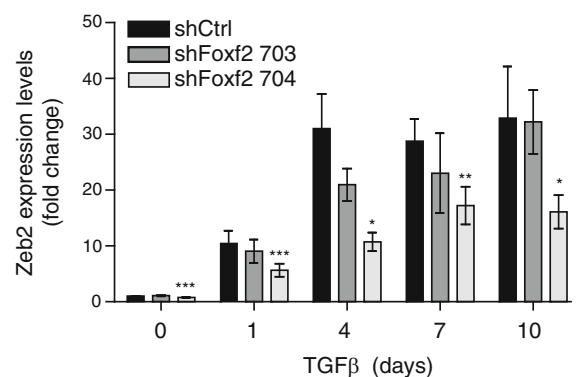

B
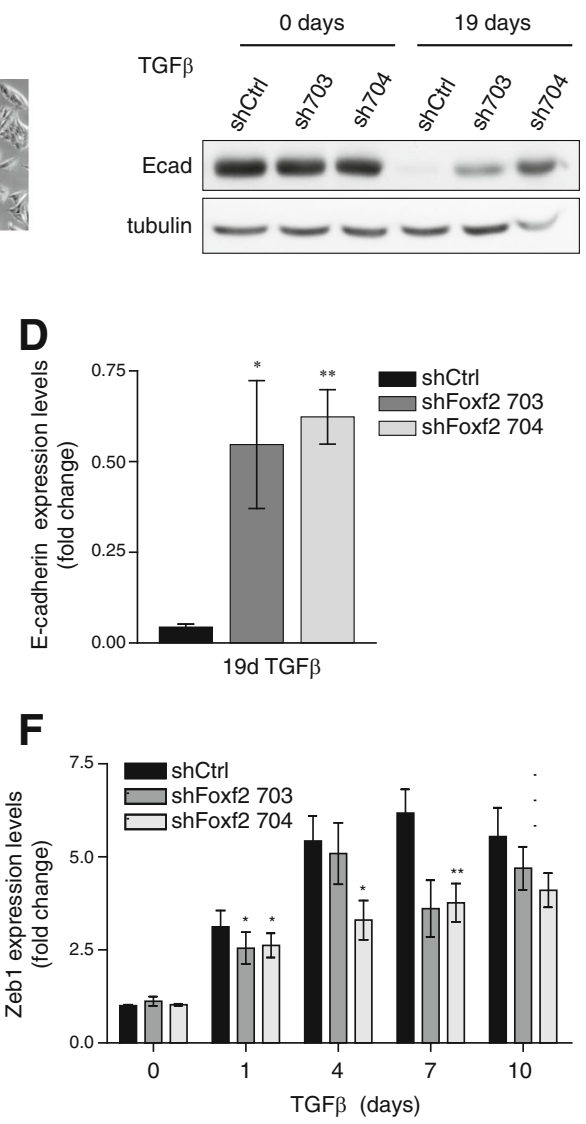

H

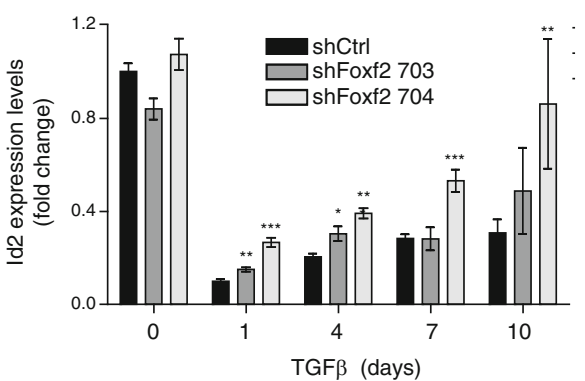

Fig. 2 Foxf2 regulates cell migration and the expression of E-cadherin transcriptional repressors. a Phase-contrast micrographs of NMuMG cells stably expressing a control shRNA (shCtrl) or a Foxf2-specific shRNA (shFoxf2 703, 704) treated with transforming growth factor (TGF) $\beta$ for 20 days. Scale bar $=100 \mu \mathrm{m}$. b shRNA-mediated ablation of Foxf2 leads to sustained E-cadherin expression after 19 days of TGF $\beta$ treatment shown by immunoblotting analysis in shFoxf2 and shCtrl NMuMG cells. Tubulin was used as a loading control. c Foxf2 and $\mathbf{d}$ E-cadherin mRNA levels were determined by quantitative RT-PCR in shFoxf2 and shCtrl NMuMG cells treated with TGF $\beta$ for 19 days. Values were normalized to RPL19 and reported as fold changes compared with untreated shCtrl NMuMG cells. e Depletion of Foxf2 leads to reduced cell migration of NMuMG cells treated for 19 days with TGF $\beta$ through transwell filters compared with control cells (shCtrl). mRNA levels of $\mathbf{f}$ Zeb1, $\mathbf{g}$ Zeb2, and $\mathbf{h}$ Id2 were determined by quantitative RT-PCR in shFoxf2- and shCtrl-transfected NMuMG cells. Values were normalized to RPL19 and reported as fold changes compared with untreated shCtrl NMuMG cells. Data are shown as mean \pm SEM of three independent experiments. Statistical values were calculated using a paired two-tailed $t$ test between shCtrl and shFoxf2 cells. ${ }^{*} p \leq 0.05$; ${ }^{* *} p \leq 0.01 ;{ }^{* * *} p \leq 0.001$ 
The loss of E-cadherin is often attributed to transcriptional dysregulation. Several transcription factors have been identified that are able to repress E-cadherin gene expression, among them the zinc-finger-homeodomain transcription factors Zeb1 and Zeb2 [55-57]. Quantitative RT-PCR analysis revealed that shRNA-mediated ablation of Foxf2 expression during a TGF $\beta$-induced EMT in NMuMG cells attenuated the upregulation of Zeb1 and Zeb2 expression observed in shCtrl-transfected cells (Fig. 2f, g). Inhibitors of differentiation (Ids) act as positive regulators of proliferation and as negative regulators of differentiation. The Id proteins lack a DNA-binding motif and inhibit, for example, E2A-dependent suppression of the E-cadherin promoter [58]. Consistent with the sustained expression of E-cadherin, ablation of Foxf2 in TGF $\beta$-treated NMuMG cells interfered with the downregulation of Id2 expression during an EMT (Fig. 2h).

The loss of Foxf2 maintained E-cadherin expression during a TGF $\beta$-induced EMT in NMuMG cells in a similar manner as the loss of the major transcriptional repressor of E-cadherin expression, Zeb1 (Additional file 1: Figure S3A, B). Also comparable to the siRNA-mediated ablation of Zeb1 expression, loss of Foxf2 expression resulted in the upregulated expression of the miR-200 family members miR-200a-3p, miR-200b-3p, and miR-429-3p (Additional file 1: Figure $\mathrm{S} 3 \mathrm{C})$. On the other hand, both Foxf2 and Zeb1 are predicted targets of miR-200 family members, and ectopic expression of the miR-200 family members miR-200b-3p, miR-200c-3p, and miR-429-3p downregulated both transcription factors (Additional file 1: Figure S3D, E). Consistent with a regulatory role of Foxf2 on the expression of Zeb family proteins, Foxf2 upregulation after 1 day of TGF $\beta$ treatment of NMuMG cells was followed by the induction of Zeb1 and Zeb2 expression, leading to a continuous downregulation of E-cadherin (Additional file 1: Figure S3F). These results indicate tight control of E-cadherin expression by a double-negative feedback loop between Foxf2 and miR-200 family members, as well as regulation of the expression of known targets of miR200 family members and transcriptional repressors of E-cadherin expression, such as Zeb1, Zeb2, and Id2. Similarly, Foxf2 is essential for the proper downregulation of E-cadherin and the regulation of Zeb2 and Id2 during a TGF $\beta$-induced EMT of Py2T murine breast cancer cells that have been derived from a tumor of the MMTV-PyMT mouse model of breast cancer (Additional file 1: Figure S3G).

In conclusion, the upregulation of Foxf2 in NMuMG cells undergoing an EMT is essential for the transcriptional repression of E-cadherin, for the disruption of cell-cell adhesions, and for EMT-associated cell migration, yet has only minor effects on the induction of mesenchymal marker expression.

\section{Foxf2 regulates cell death and survival pathways}

To identify the actual genes and signaling pathways that are regulated by Foxf2 during an EMT, we performed gene expression profiling by RNA sequencing of NMuMG cells that were transfected with control siRNA (siCtrl; epithelial state) or with siCtrl or siRNA targeting Foxf2 (siFoxf2) in the presence of TGF $\beta$ for 4 days (mesenchymal state). We found 1789 genes to be differentially expressed at least twofold upon Foxf2 knockdown compared with siRNA control at 4 days of TGF $\beta$ treatment, and 2689 genes were significantly changed upon induction of EMT comparing siCtrl in the absence or presence of TGF $\beta$ (siCtrl 0 days vs 4 days TGF $\beta$; EMT). In total, 792 genes were commonly regulated by the loss of Foxf 2 expression and by the induction of an EMT with TGF $\beta$ (Fig. 3a; Additional file 1: Table S1). Unsupervised hierarchical clustering revealed that Foxf2-deficient cells treated with TGF $\beta$ more closely resembled the mesenchymal state of TGF $\beta$-treated control cells, however they formed a separate clustering arm (Fig. 3b). To study in more detail which transcripts were specifically altered compared with the mesenchymal and epithelial control states, gene expression signatures were generated using weighted gene coexpression network analysis (WGCNA). Six different gene expression signatures were extracted, with the yellow and the brown signatures summarizing genes from an intermediate mesenchymal state (Fig. 3b; Additional file 1: Table S2). The EMT-induced expression of the genes in the yellow signature was strongly reduced by the ablation of Foxf2 knockdown. Conversely, the EMT-repressed expression of the genes in the brown signature was blocked by the loss of Foxf2 (Fig. 3c).

Pathway enrichment analysis using ingenuity pathway analysis (IPA) revealed major functions of the yellow signature-associated genes in cellular movement and cell-cell signaling and interaction, functions that can be attributed to the loss of E-cadherin expression as described above (Fig. 3d). On the other hand, the brown signature was found to be associated with molecular transport and metabolism (lipid, vitamin, and mineral metabolism) pathways (Fig. 3d). Interestingly, both upand downregulated EMT signatures that are also affected by Foxf2 knockdown (brown and yellow signatures) were enriched in pathways describing cell death and survival, indicating a regulatory role of Foxf2 in these processes (Fig. 3d). Indeed, NMuMG cells stably expressing shRNA against Foxf2 showed significantly less TGF $\beta$-mediated growth inhibition compared with shCtrl-transfected cells, and the cell number increased significantly compared with shCtrl-expressing cells (Fig. 4a).

Foxf 2 affects apoptosis by regulating the expression of Noxa To assess whether the increase in cell numbers was due to increased proliferation or decreased cell death, we 
A

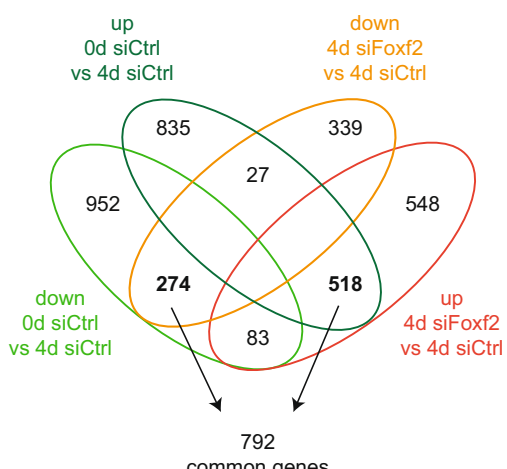

common genes
B

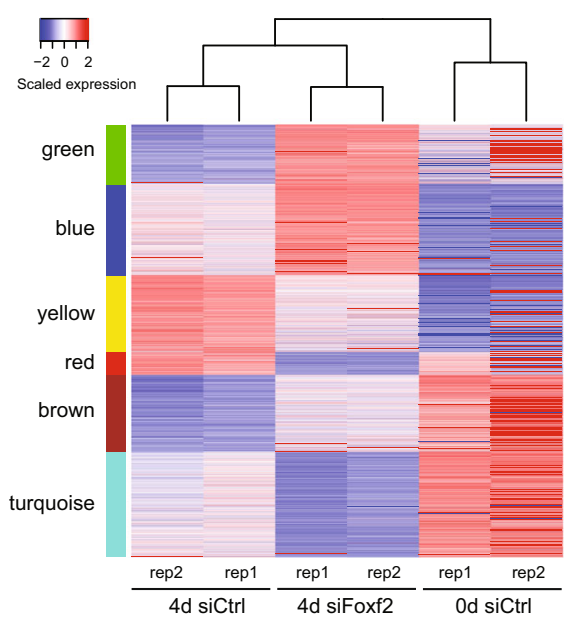

C
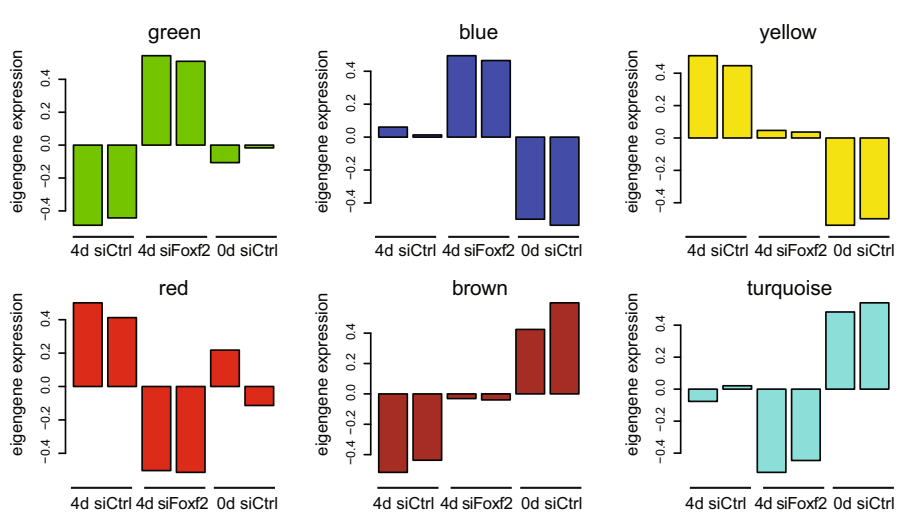

D
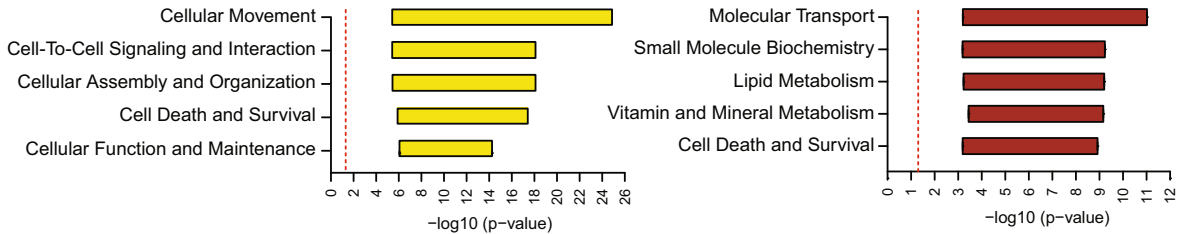

Fig. 3 RNA sequencing analysis of Foxf2-dependent gene expression. a Venn diagram illustrating the overlap of significantly differentially expressed genes ( $p$ adjusted $<0.05$, abs (log2 fold change) $\geq 1$ ) between the epithelial and mesenchymal state ( 0 days vs 4 days siRNA control (siCtrl)) or for the Foxf2 perturbation ( 4 days siFoxf2 vs 4 days siCtrl). Highlighted in bold are genes whose change during EMT is reversed by Foxf2 knockdown.

b Heatmap of gene expression from RNA-sequencing of epithelial control samples (untreated siCtrl NMuMG cells) and of siCtrl- or siFoxf2-treated NMuMG cells in the presence of TGF $\beta$ for 4 days. The sample order in the heatmap was obtained from an unsupervised hierarchical clustering, while rows (genes) were arranged according to the gene signatures derived from WGCNA. $\mathbf{c}$ The eigengene expression of the different gene signatures derived from WGCNA (see a) illustrates a general expression trend of all genes belonging to a gene signature. $\mathbf{d}$ IPA analysis of the brown and yellow gene signature. Shown in the bar plot are the significance ranges of pathways belonging to the most significantly enriched categories. The dotted red line indicates a $p$ value of 0.05. Differential gene expression and gene signature memberships are reported in Additional file 1 (Table S1 and S2, respectively)

compared the rates of apoptosis and proliferation by Annexin- $\mathrm{V}$ staining and BrdU incorporation and PI staining, respectively, in NMuMG cells treated with TGF $\beta$ and depleted or not for Foxf2 expression. The loss of Foxf2 significantly reduced the levels of apoptosis when compared with control cells (Fig. 4b), while only a moderate difference in the number of cycling cells was observed (Additional file 1: Figure S4A). The lack of Foxf2 reduced caspase-dependent programmed cell death, as the levels of cleaved caspase- 3 and its downstream cleavage target poly-(ADP-ribose) polymerase (PARP) were diminished upon knockdown of Foxf2 (Fig. 4c). In summary, the results show that the increased expression of Foxf2 during EMT is critical for promoting TGF $\beta$-induced cell death.

TGF $\beta$ has been shown to act as a tumor suppressor in the early stages of tumorigenesis by inducing the expression of cell cycle inhibitors and proapoptotic factors 

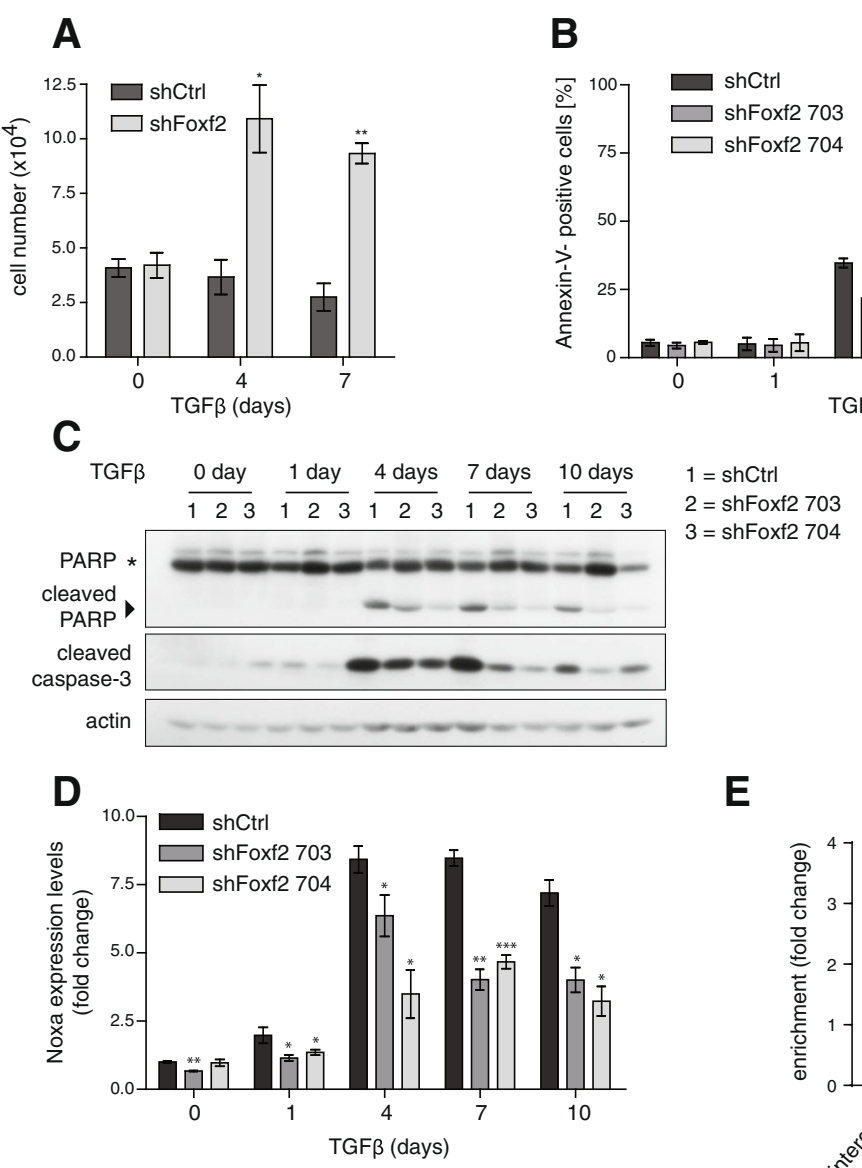

E

$\mathbf{F}$

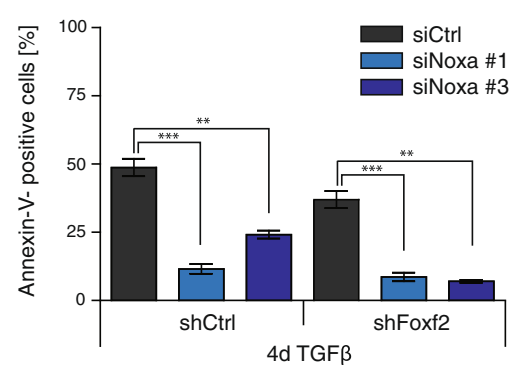

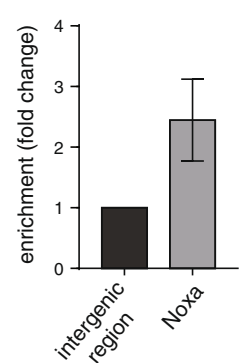

G

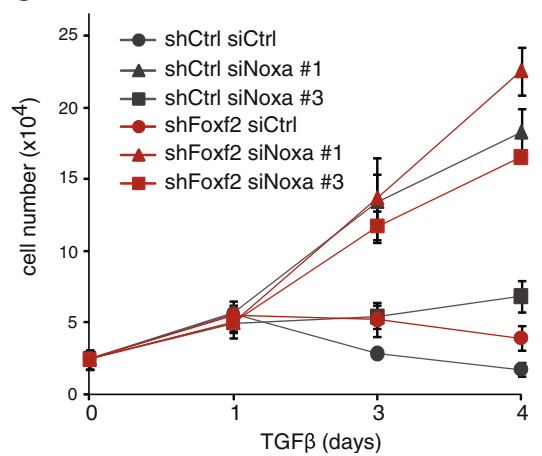

Fig. 4 (See legend on next page.) 


\begin{abstract}
(See figure on previous page.)
Fig. 4 Depletion of Foxf2 attenuates TGFß-induced apoptosis and the expression of the proapoptotic protein Noxa. a Downregulation of Foxf2 promotes cell proliferation. shFoxf2- and shCtrl-expressing NMUMG cells were treated with transforming growth factor (TGF) $\beta$ for the times indicated and counted using a Neubauer chamber. b Foxf2 depletion decreases apoptosis during TGF $\beta$-induced EMT. shFoxf2- and shCtrl-expressing NMuMG cells were treated with TGF $\beta$ for the times indicated, and apoptosis was detected by Annexin- $V$ staining and flow cytometry analysis. c TGF $\beta$ induces classical caspase-mediated apoptosis dependent on the upregulation of Foxf2. Immunoblotting analyses of the same experiment as shown in Fig. $1 \mathrm{~d}$ for cleaved caspase-3 and PARP in shFoxf2- and shCtrl-expressing NMUMG cells treated with TGF $\beta$ for the times indicated. Actin was used as a loading control. $\mathbf{d}$ Knockdown of Foxf2 attenuates the upregulation of Noxa expression. Noxa mRNA levels in shFoxf2- and shCtrl-expressing NMuMG cells treated with TGF $\beta$ for the times indicated were determined by quantitative RT-PCR. Values were normalized to RPL19 and presented as fold changes compared with untreated shCtrl NMuMG cells. e Foxf2 regulates Noxa expression by direct transcriptional activation. Chromatin immunoprecipitation of HA-tagged Foxf2 was performed either on Foxf2-expressing or control NMuMG cells treated for 2 days with TGF $\beta$. Immunoprecipitated DNA fragments were quantified by quantitative PCR using primers covering base pairs -696 to -499 of the noxa promoter region. Enrichment (IP/input) for specific primers was calculated relative to primers covering an intergenic region. $\mathbf{f}$ Noxa depletion significantly decreases TGF $\beta$-induced apoptosis. shFoxf2- and shCtrl-expressing NMuMG cells were transfected with control siRNA (siCtrl) and two different siRNAs specific for murine Noxa (siNoxa \#1, siNoxa \#3) and incubated with TGF $\beta$ for 4 days. The extent of apoptosis was measured by Annexin-V staining and flow cytometry. $\mathbf{g}$ The impairment of Noxa expression leads to increased cell proliferation. shFoxf2- and shCtrl-expressing NMuMG cells were transfected with control siRNA (siCtrl) and two different siRNAs specific for murine Noxa (siNoxa \#1, siNoxa \#3) and incubated with TGF $\beta$ for the times indicated. Cell numbers were determined using a Neubauer chamber. Results show the mean \pm SEM of three independent experiments. Statistical values were calculated using paired/unpaired two-tailed $t$ test. ${ }^{*} p \leq 0.05 ;{ }^{* *} p \leq 0.01 ;{ }^{* * *} p \leq 0.001$
\end{abstract}

[59]. Differential gene expression analysis between TGF $\beta$-treated control and Foxf2-deficient NMuMG cells revealed a substantial regulation of the $\mathrm{BH} 3$-only factor Noxa by Foxf2, which was confirmed by quantitative RT-PCR analysis (Fig. 4d). ChIP experiments with NMuMG cells expressing HA-tagged Foxf2 treated for 2 days with TGF $\beta$ demonstrated a weak direct binding of Foxf2 to the noxa gene promoter, suggesting additional indirect regulatory mechanisms (Fig. 4e). As the upregulation of Foxf 2 is necessary for TGF $\beta$-induced Noxa expression, we next assessed whether loss of Noxa is sufficient to prevent apoptosis in TGF $\beta$-treated NMuMG cells. Noxa expression in NMuMG cells was ablated by transient transfection of two different siRNAs (siNoxa \#1, siNoxa \#3) or control siRNA (siCtrl) in shFoxf2- and shCtrl-expressing cells. Following the reduction of Noxa mRNA levels in siNoxa \#1- and \#3-treated cells (Additional file 1: Figure S4B), apoptosis was attenuated and cell growth inhibition was compensated in TGF $\beta$-treated cells (Fig. 4f, g; Additional file 1: Figure S4C). These results demonstrate that depletion of Noxa is sufficient to protect NMuMG cells from TGF $\beta$-induced apoptosis. Together, these data indicate that Foxf2 mediates TGF $\beta$-induced apoptosis by the transcriptional activation of the proapoptotic protein Noxa.

\section{Foxf2 promotes TGF $\beta$-induced growth arrest by repressing EGF receptor signaling}

As well as activating transcription of the noxa gene and inducing apoptosis, we investigated whether Foxf2 regulates any prosurvival signaling pathway. EGF receptor (EGFR) family members are known to provide protection from TGF $\beta$-induced cell cycle arrest and apoptosis by activating the PI3K pathway $[60,61]$. Indeed, immunoblotting analyses revealed that the levels of activated (tyrosine 1173-phosphorylated) forms of EGFR were higher in
Foxf2-depleted NMuMG cells compared with control NMuMG cells when treated with TGF $\beta$ (Fig. 5a).

Previously, we have reported that survival of NMuMG cells undergoing a TGF $\beta$-induced EMT depends on activated EGFR signaling [20]. We thus investigated whether inhibition of EGFR signaling impaired the antiapoptotic effect of Foxf2 depletion. Towards this aim, shFoxf2- and shCtrl-transfected NMuMG cells were treated with the EGFR inhibitor (EGFRi) AG1478 during TGF $\beta$ treatment, and cell growth and rates of apoptosis were determined. Combined treatment with EGFRi and TGF $\beta$ for 4 days led to a significantly reduced growth in shFoxf2-expressing NMuMG cells compared with the solvent (dimethyl sulfoxide (DMSO))-treated shFoxf2-expressing NMuMG cells (Fig. 5b; Additional file 1: Figure S5A). In addition, treatment of shFoxf2-expressing NMuMG cells with AG1478 increased apoptosis to a similar extent as observed in shCtrl-expressing cells (Fig. 5c). The extent of apoptosis thereby correlated with the levels of EGFR inhibition (Fig. 5d). This result indicates that TGF $\beta$-resistant growth of Foxf2 knockdown cells relies on the activation of EGFR survival signaling.

To investigate how Foxf2 influences EGFR activation, we assessed whether the expression of EGFR ligands was affected by the modulation of Foxf2 expression. Gene expression profiling and validation by quantitative RT-PCR revealed that the EGFR-ligands betacellulin (Btc), amphiregulin (Areg), and (moderately) epiregulin (Ereg) showed sustained expression upon knockdown of Foxf2 during an EMT in both NMuMG and Py2T cells (Additional file 1: Figure S5B, C). Promoter binding prediction programs indicated a potential direct binding of Foxf2 to the Btc promoter (data not shown). ChIP followed by quantitative PCR of HA-tagged Foxf2 in NMuMG cells during TGF $\beta$-induced EMT revealed a direct binding of Foxf2 to the Btc promoter region, to a 
A

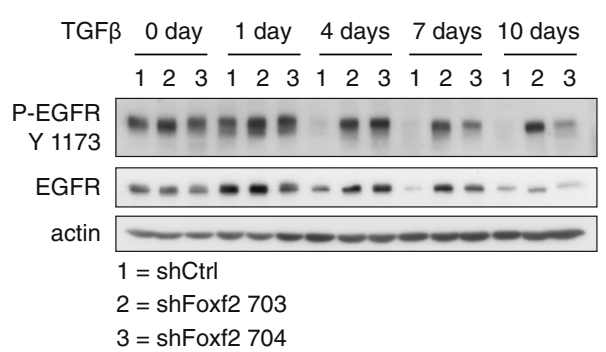

C

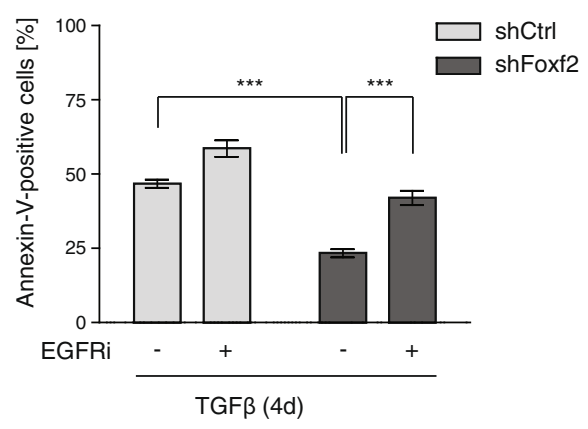

B - shCtrl + DMSO

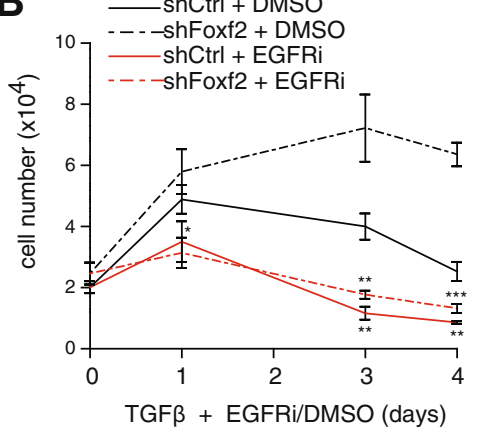

D

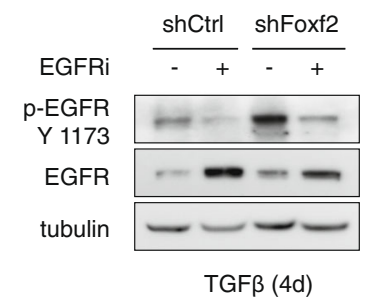

E

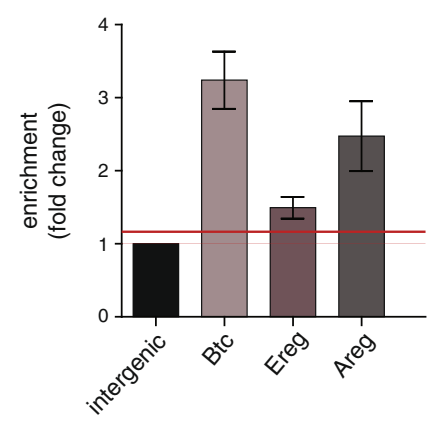

$\mathbf{F}$

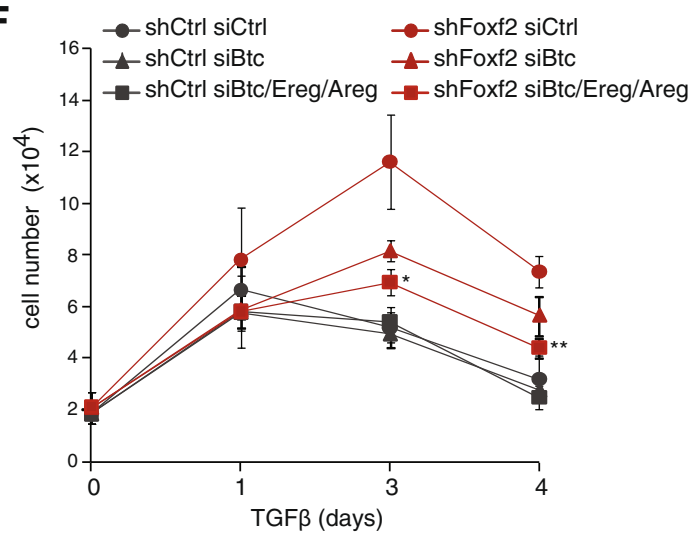

Fig. 5 Inhibition of EGFR signaling increases apoptosis in Foxf2-depleted cells. a Depletion of Foxf2 leads to sustained epidermal growth factor receptor (EGFR) activation. Immunoblotting analysis of the phosphorylation status of EGFR and total EGFR protein levels in shFoxf2- and shCtrl-expressing NMuMG cells treated with transforming growth factor (TGF) $\beta$ for the times indicated. Actin was used as a loading control. b-d NMUMG cells expressing shRNA specific for Foxf2 or control shRNA were treated with TGF $\beta$ and AG1478 (EGFR inhibitor (EGFRi)) or control solvent (dimethyl sulfoxide (DMSO)) for the indicated times. b Cell numbers were determined using a Neubauer chamber. $\mathbf{c}$ EGFR inhibition significantly increases apoptosis in Foxf2 knockdown cells. Apoptosis was detected by Annexin-V staining and flow cytometry. d Treatment with AG1478 decreases EGFR activation in shFoxf2 cells to a similar extent as seen in TGF $\beta$-treated NMuMG cells expressing control shRNA. Immunoblotting analysis for EGFR phosphorylation and total EGFR levels is shown. Tubulin was used as a loading control. e Foxf2 regulates the expression of EGFR ligands by direct transcriptional repression. Chromatin immunoprecipitation of Foxf2 was performed either on HA-Foxf2 expressing or control NMUMG cells treated for 2 days with TGF $\beta$. Immunoprecipitated DNA fragments were quantified by quantitative PCR using primers covering base pairs -450 to -253 of the Btc promoter region, base pairs -851 to -654 of the Ereg promoter, and base pairs +1086 to 1210 of the Areg exon 2. Enrichment (IP/input) for specific primers was calculated relative to primers covering an intergenic region. $\mathbf{f}$ Individual depletion of Btc or combined depletion of betacellulin (Btc), epiregulin (Ereg), and amphiregulin (Areg) reduces cell numbers in shFoxf2- but not in shCtrl-expressing NMuMG cells in the presence of TGF $\beta$ for 4 days. Cell numbers were determined using a Neubauer chamber. Data are shown as mean \pm SEM of at least three independent experiments. Statistical values were calculated using a paired/unpaired two-tailed $t$ test. ${ }^{*} p \leq 0.05 ;{ }^{* *} p \leq 0.01 ;{ }^{* *} p \leq 0.001$ 
regulatory region in exon 2 of the Areg gene, and with less efficiency to the Ereg promoter region (Fig. 5e).

To assess whether Btc, Areg, or Ereg were responsible for the stimulation of EGFR and increased cell survival of Foxf2-depleted cells, NMuMG cells stably expressing shRNA against Foxf2 or a control shRNA were transiently transfected with siRNAs against Btc or with a mix of siRNAs against Btc, Areg, and Ereg and treated with TGF $\beta$. The efficiency of Btc or combined Btc/Areg/Ereg ablation was determined by quantitative RT-PCR (Additional file 1: Figure S5D, E). Knockdown of Btc alone or in combination with the other two family members resulted in reduced cell growth in shFoxf2-expressing cells when treated with TGF $\beta$ (Fig. 5f). These results indicate that attenuation of EGFR activation by siRNA-mediated depletion of its ligands abrogates the survival benefit of Foxf2 depletion. We conclude that TGF $\beta$-induced Foxf2 expression represses the transcriptional activation of the Btc and Areg genes, resulting into a reduced expression of these EGFR ligands and a repression of EGFR survival signaling.

Foxf2 expression correlates with poor prognosis in patients Cancer-associated gene expression profiling has emerged as an appropriate tool to predict the relapse risk and to identify genes that mediate disease progression. To investigate whether Foxf2 expression is predictive for tumor progression or metastasis formation, we analyzed a breast cancer database of the Memorial Sloan-Kettering Cancer Center (MSKCC), published by Minn et al. [62]. This "Minn" database consists of microarray gene expression analysis of tumor samples from 82 patients with advanced breast cancer (T2-T4). The tumors were divided into two groups according to the log expression levels relative to the median expression of the investigated gene. The low and high Foxf2-expressing groups were further stratified for lymph node (LN) metastasis status. Interestingly, low Foxf2 expression significantly correlated with early distant metastasis formation in lymph node-negative $\left(\mathrm{LN}^{-}\right)$tumors, whereas the opposite tendency was found in tumors of patients that were positive for lymph node metastasis $\left(\mathrm{LN}^{+}\right)$(Fig. 6a, b).

To further substantiate a potential correlation between Foxf2 expression and patient survival, we analyzed the Netherlands Cancer Institute (NKI295) breast cancer database for Foxf2 expression [63]. The NKI295 database consists of microarray gene expression analysis of tumor samples from 295 patients with early-stage breast cancer (stage I or stage II primary breast carcinomas). Although Foxf2 expression was not predictive for metastasis formation or survival in the total patient pool (Fig. 6c), high expression of Foxf2 correlated with poor overall survival in patients with luminal subtype B breast cancer (Fig. 6d). High expression of Foxf2 in tumors with negative estrogen receptor (ER) status correlated with high significance of early metastasis onset as well as poor overall survival (Fig. 6e, f). Similarly, in a large tumor collection from the Metabric consortium [64, 65], high Foxf2 expression predicted worse survival in the luminal B breast cancer subtype (Fig. 6g). Interestingly, Foxf2 expression was significantly higher in more aggressive tumor subtypes, such as $\mathrm{ER}^{-}$compared with $\mathrm{ER}^{+}$, triple-negative compared with all other subtypes, and in claudin-low tumors (the breast cancer subtype associated with an EMT signature), compared with all others (Fig. 6h).

Together, the expression of Foxf2 in human patient samples and its prediction for clinical outcome reflect the dual function of Foxf2 observed in our in-vitro studies. Foxf 2 may function as a tumor suppressor in early cancer development by promoting apoptosis, hence showing a poor prognosis in $\mathrm{LN}^{-}$patients with low Foxf2 expression. More advanced tumors with high Foxf2 expression correlate with shorter metastasis-free survival, supporting a role of Foxf2 in cancer cell invasion and metastasis formation.

\section{Discussion}

Overcoming the growth inhibitory effect of TGF $\beta$ during the early stages of tumorigenesis as well as the conversion of TGF $\beta$-mediated growth inhibition into TGF $\beta$-induced tumor progression are fundamental processes during primary tumor growth and metastasis formation $[1,66]$. Thus, understanding the mechanisms underlying this dual role of TGF $\beta$ in cancer progression and the strategies of cancer cells to circumvent TGF $\beta$-induced apoptosis may offer new opportunities for novel cancer therapies.

Here, we have employed nontransformed NMuMG cells and Py2T murine breast cancer cells to delineate the molecular mechanisms underlying a TGF $\beta$-induced EMT, including overcoming TGF $\beta$-induced resistance to apoptosis and the acquisition of invasive properties. We report a dual function of the transcription factor Foxf2, acting as a tumor suppressor by promoting apoptosis and by repressing survival, while exerting protumorigenic activity at later stages of tumor progression, such as a promigratory function by inducing the disruption of cell-cell adhesion. Foxf2 is upregulated via the canonical TGF $\beta$ pathway, and gain of function studies in NMuMG cells reveal that its expression is not sufficient to induce an EMT. However, loss of function studies demonstrate that Foxf2 is essential for the disruption of cell junctions and the repression of epithelial marker expression but not for the gain of mesenchymal marker expression. Notably, Foxf 2 is crucial for TGF $\beta$-mediated cell death by the transcriptional activation of the Noxa gene, encoding for a BH3-only proapoptotic factor, and the subsequent induction of caspase-dependent apoptosis. Moreover, Foxf2 directly represses transcription of the Btc 


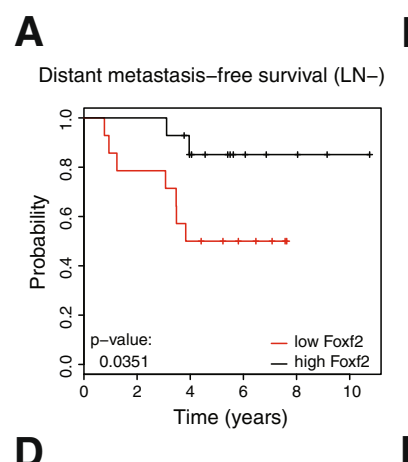

D

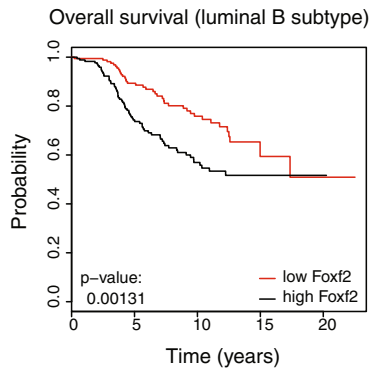

G

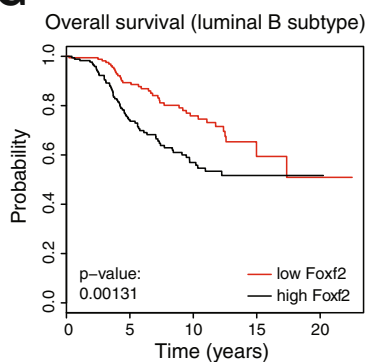

B

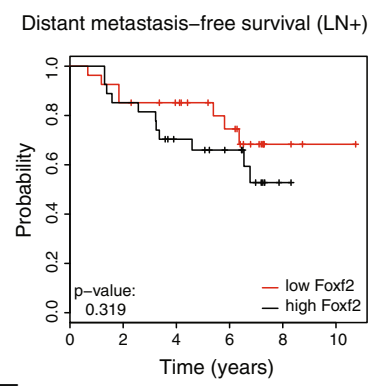

E

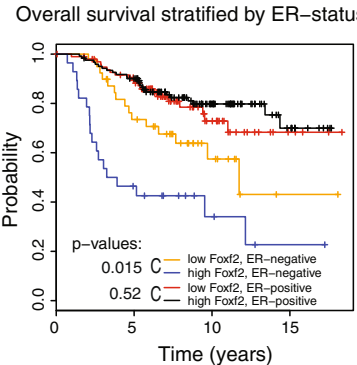

$\mathbf{F}$
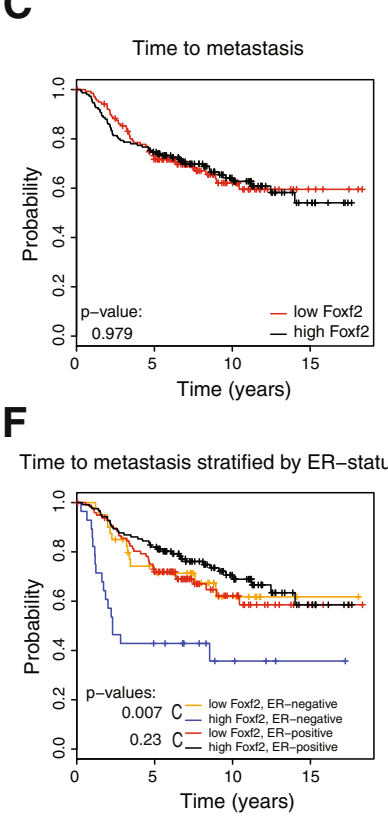

H

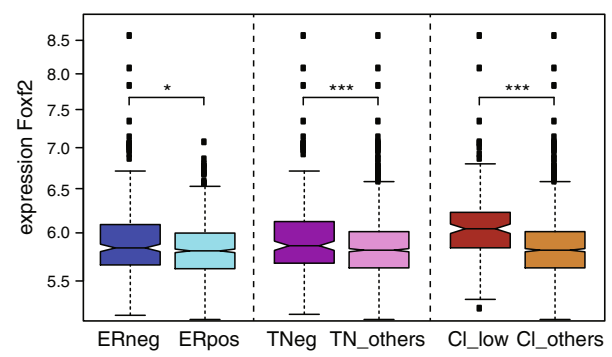

Fig. 6 High Foxf2 expression correlates with good prognosis in early-onset breast cancer patients but with poor prognosis in late-stage estrogen receptor-negative and luminal B breast cancer patients. a,b Statistical analysis of the Memorial Sloan-Kettering Cancer Center (Minn) database. Tumors were divided into high and low Foxf2-expressing groups based on the median expression of Foxf2 mRNA. Expression of Foxf2 was correlated with distant metastasis free survival in lymph node-negative $\left(\mathrm{LN}^{-}\right)$tumors $(n=28)$ or $\mathrm{LN}^{+}$tumors $(n=54)$. Foxf2 expression is predictive for metastasis incidence in $L N^{-}$tumors (a), where low Foxf2 expression correlates with early metastases onset ( $p$ value $=0.0351$ ), but not for $L N^{+}$ tumors (b). c-f Statistical analysis of the Netherlands Cancer Institute (NKI295) database. Tumors were divided into high and low Foxf2-expressing groups based on the relative expression of Foxf2 compared with the tumor pool (log fold change $=0$ ). Expression of Foxf2 was correlated with time to metastasis $(\mathbf{c}, \mathbf{f})$ or overall survival $(\mathbf{d}, \mathbf{e})$ either in all tumors analyzed $(\mathbf{c} ; n=288)$ or stratified for estrogen receptor (ER) expression (e, $\mathbf{f}$; ER-negative: $n=68$; ER-positive: $n=220$ ). Foxf2 expression does not correlate with tumor metastasis in the total tumor pool (c), but high Foxf2 expression is predictive for overall patient survival in luminal subtype $\mathrm{B}(\mathbf{d} ; p=0.00131)$ and ER-negative tumors (e; $p=0.0154)$, respectively. High Foxf2 expression levels also correlate with an early onset of metastasis in ER-negative patients $(\mathbf{f} ; p=0.007)$. $\mathbf{g}$, $\mathbf{h}$ Statistical analysis of the Metabric database ( $n=1298$ tumors). $\mathbf{g}$ Tumors were divided into high and low Foxf2-expressing groups based on the median expression of Foxf2 mRNA. In the luminal subtype B tumors $(n=352)$, Foxf2 expression is predictive for overall survival ( $p$ value of the likelihood-ratio test). $\mathbf{h}$ Expression of Foxf2 across multiple different tumor subtypes shows significantly increased levels in ER-negative (ERneg) vs ER-positive (ERpos) tumors, in triple negative (TNeg), and in claudin-low (Cl_low) compared with all other tumors ( $p$ value of the Kruskal-Wallis test; ${ }^{*} p \leq 0.05$; ${ }^{* * *} p \leq 0.001$ )

and Areg genes, encoding for ligands of EGFR, and thus attenuates EGFR-mediated survival signaling (Fig. 7).

The failure of Foxf2-depleted cells to disrupt tight and adherens junctions exemplifies its indispensable role in the acquisition of an invasive cell morphology. Loss of E-cadherin is an early event during EMT resulting in the disruption of the polarity complex, a prerequisite for the dissociation and invasion of cancer cells [67-69]. Direct transcriptional repression has emerged as one common regulatory mechanism of E-cadherin expression in various cancer types [70, 71]. Here we demonstrate that, by mediating the TGF $\beta$-induced upregulation of the transcriptional repressors Zeb1 and Zeb2 as well as the repression of Id2, Foxf2 mediates the transcriptional downregulation of E-cadherin and consequently the disruption of cell-cell adhesion. In addition, we show that 


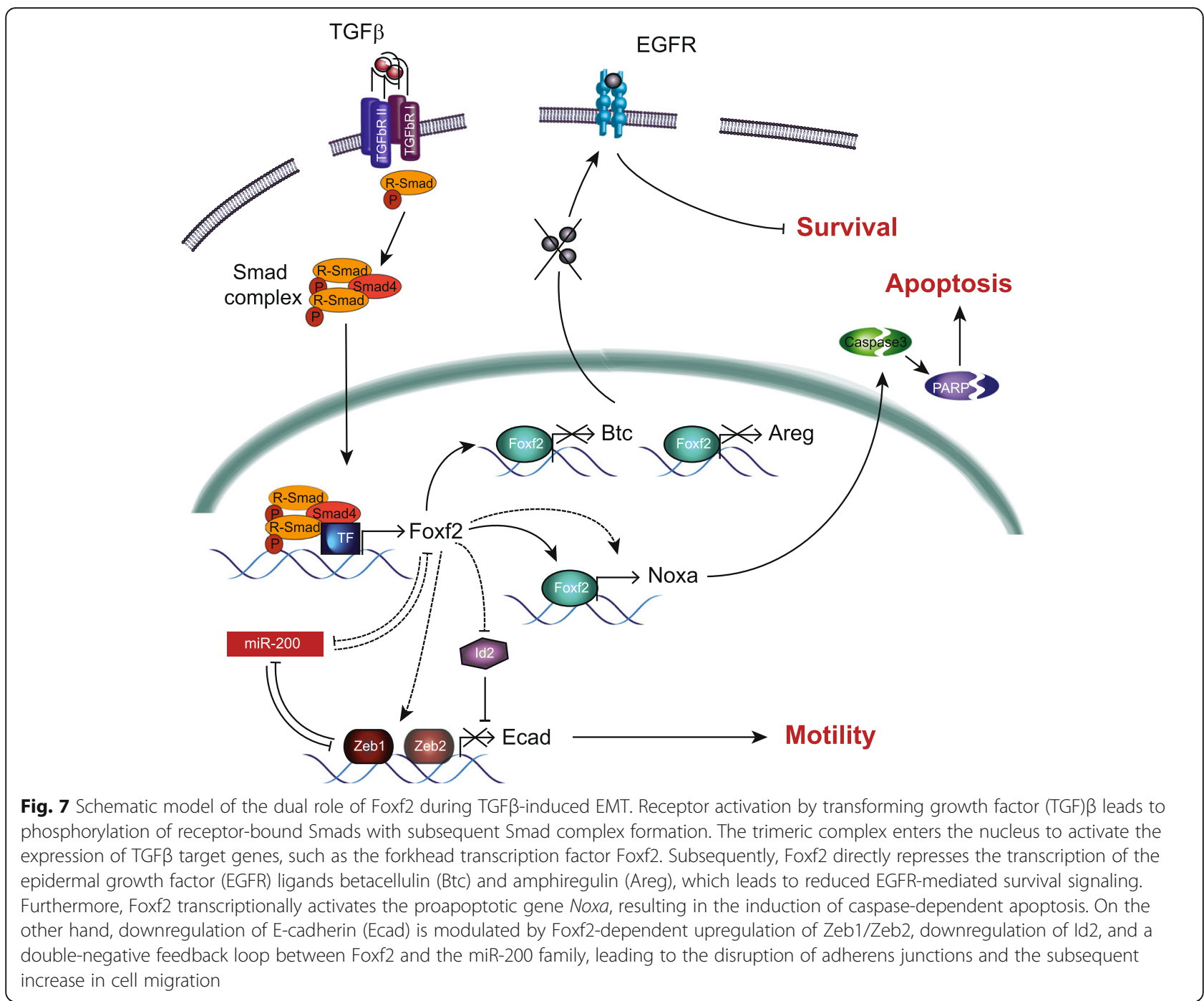

Foxf2 also affects the expression of the miR-200 family which are potent EMT-repressing noncoding RNAs that target Zeb1 and Zeb2 transcripts [72-74]. Interestingly, the expression of Foxf2 and miR-200 are controlled in a double-negative feedback loop, similar to the well-studied Zeb1-miR200 loop. The mechanism by which Foxf2 regulates the expression of these transcription (co)factors remains elusive, but the presence of putative Foxf2 binding sites in the promoter region of Zeb1 and Zeb2 (data not shown) and data from Kundu et al. [75] in nonsmall-cell lung cancer (NSCLC) cells suggest a direct regulatory mechanism, thus ensuring the downregulation of cell-cell junctions at multiple levels (Fig. 7). Moreover, consistent with our finding that Foxf2 predicts poor survival in a subset of breast cancer patients, our results identify Foxf2 as a promigratory and prometastatic factor (Fig. 7).
Our results also show that Foxf2 is essential for TGF $\beta$-mediated apoptosis. The reduction of TGF $\beta$-induced apoptosis in Foxf2-deficient cells is a consequence of the loss of the transcriptional activation of Noxa gene expression. Noxa has been shown to be critical in fine-tuning cell death decisions via degradation of the prosurvival molecule Mcl1 [76]. Noxa has been identified as a primary p53 target gene; however, oncogenic stress, such as irradiation and hypoxia, results in efficient induction of Noxa also in the absence of p53 [77, 78]. Our findings identify Foxf2 as a novel transcriptional activator of the tumor suppressor Noxa (Fig. 7).

Besides triggering apoptosis via regulation of Noxa expression, Foxf2 is also involved in the negative regulation of survival signals by the transcriptional repression of the EGFR ligands betacellulin (Btc), amphiregulin (Areg), and, to a lesser degree, epiregulin (Ereg). Although the regulatory effect of Foxf2 on the transcription level of 
the individual EGFR ligands is moderate (Additional file 1: Figure S5B, C), a cumulative effect by the simultaneous modulation of Btc, Areg, and Ereg expression enables a significant shift towards less EGFR signaling (Fig. 5a). Reduced EGFR signaling leads to a reduction in EGFR phosphorylation and, hence, reduced PKB activation. Blocking EGFR signaling has been shown to amplify the apoptotic response to TGF $\beta$ [61]. Here, we show that both pharmacological receptor inhibition as well as the combined reduction of the expression of the EGFR ligands Btc, Areg, and Ereg increased TGF $\beta$-induced apoptosis in Foxf2-depleted NMuMG cells. These results indicate that, in addition to Noxa regulation, Foxf2 mediates its apoptotic effect by blocking EGFR-mediated survival signaling (Fig. 7).

To support the importance of a Foxf2 function during tumor development and progression, we have performed correlation studies for Foxf2 on three different breast cancer databases [62-64]. Analysis of a lymph node-negative stratified patient subset demonstrates that low Foxf2 expression significantly correlates with early metastasis formation. Comparably, low Foxf2 expression has been recently reported to correlate with early-onset metastasis and poor prognosis in breast cancer patients [43]. Interestingly, the opposite is found in more progressive breast cancers, where high Foxf2 expression correlates with poor prognosis. Stratification for luminal subtype B or for ER status reveals a highly significant correlation of high Foxf2 expression and early metastasis onset, concomitant with reduced overall survival. $\mathrm{ER}^{-}$tumors represent highly aggressive breast cancer subtypes. In addition, Foxf2 transcript levels are increased in more aggressive $\mathrm{ER}^{-}$, in triple negative, and in the EMT-like, claudin-low breast cancer subtypes. Together, our findings identify high levels of Foxf2 as a marker for good prognosis in early noninvasive stages of tumor development, but with poor prognosis in malignant stages [79]. These findings substantiate the dual role of Foxf2 in cancer patients.

\section{Conclusions}

In our study, we demonstrate a dual role for the transcription factor Foxf2. It induces proapoptotic and represses antiapoptotic genes and, thus, acts as a tumor suppressor, likely with the help of specific cofactors. On the other hand, it induces the expression of EMT-inducing genes and thus exerts prometastatic functions to cells that have overcome the apoptotic crisis and undergone EMT. The role of Foxf2 in pre- and post-EMT cells reflects the well-studied dual role of TGF $\beta$ in cancer progression. Our results also substantiate findings in knockout mouse models where Foxf2 was found to play an important role in EMT-associated developmental processes and maintenance of the epithelial-mesenchymal structure in lung and gut tissues
[38, 39]. Hence, fine-tuning of the expression of Foxf2 and its cofactors could be pivotal during carcinogenesis, and insights into its regulation and molecular function are critical for the design of novel therapeutic strategies.

\section{Additional file}

\begin{abstract}
Additional file 1: Figure S1. Foxf2 expression is upregulated during EMT via the canonical Smad pathway. The increased expression of Foxf2 during an EMT was assessed in normal murine mammary gland epithelial cells (NMuMG) and in murine and human breast cancer cells. Figure S2. Foxf2 is required for TGF $\beta$-induced disruption of adherens junctions. RNAi-mediated ablation prevents an EMT as visualized by immunofluorescence staining for epithelial and mesenchymal markers. Figure S3. Foxf2 regulates the expression of Zeb1, Zeb2, Id2, and members of the miR-200 family as determined by quantitative RT-PCR of their expression during an EMT in the absence of presence of Foxf2. Figure S4. Foxf2 regulates Noxa expression and thus affects cell proliferation and apoptosis. Foxf2 regulated the expression of Noxa, and siRNA-mediated depletion of Noxa prevented an increase in cell death induced by the loss of Foxf2 expression as assessed by quantitative RT-PCR. Figure S5. EGF ligandmediated EGF receptor signaling overcomes Foxf2-controlled cell survival. Foxf represses the expression of EGF receptor ligands as assessed by quantitative RT-PCR. Supplementary material and methods. Detailed information is given on the antibodies and reagents, on biochemical and cell biological methods, and on RNA sequencing and bioinformatics analysis used in the study. Table S1. Excel file summarizing the differential expression analysis (siFoxf2 to siCtrl after 4 days TGF $\beta$ treatment or siCtrl with vs without TGF $\beta$ for 4 days) of all transcripts detected with RNA-sequencing. Table S2. Excel file showing the list of genes belonging to the different gene signatures (modules) and the strength of their modular membership (kME values). (ZIP $14675 \mathrm{~kb}$ )
\end{abstract}

\section{Abbreviations \\ Areg: Amphiregulin; Btc: Betacellulin; ChIP: Chromatin immunoprecipitation; EGFR: Epidermal growth factor receptor; EGFRi: EGFR inhibitor; EMT: Epithelial to mesenchymal transition; Ereg: Epiregulin; Foxf2: Forkhead box protein F2; Ids: Inhibitors of differentiation; IPA: Ingenuity pathway analysis; MET: Mesenchymal to epithelial transition; NMuMG: Normal murine mammary gland; PARP: Poly-(ADP-ribose) polymerase; TGF: Transforming growth factor; WGCNA: Weighted gene coexpression network analysis}

\section{Acknowledgments}

We thank Drs. L. Lundh, M. Kaeser, and P. ten Dijke for sharing important reagents. We are grateful to P. Schmidt, H. Antoniadis, I. Galm, U. Schmieder, and R. Jost for excellent technical support. We thank P. Lorentz and the DBM microscopy facility (DBM, University of Basel) for excellent support with microscopy, C. Beisel and the Genomics Facility Basel (D-BSSE) for next generation RNA sequencing, and R. Ivanek and the Bioinformatics Core Facility (DBM, University of Basel) for help with RNA sequencing analysis.

\section{Funding}

This research has been supported by the Swiss National Science Foundation, the Swiss Initiative for Systems Biology (RTD Cellplasticity), and the Swiss Cancer League. NM-S was supported by a Marie-Heim Vögtlin grant from the Swiss National Foundation.

\section{Availability of data and materials}

The RNA expression data from the RNA-sequencing are deposited at Gene Expression Omnibus (https://www.ncbi.nlm.nih.gov/geo/; GEO accession number: GSE112796).

\section{Authors' contributions}

NM-S, CH, ST, and MY designed and performed the experiments, analyzed the data, and wrote the manuscript. GC designed the experiments, analyzed the data, and wrote the manuscript. All authors read and approved the final manuscript. 


\section{Ethics approval and consent to participate}

In this work, no experiments have been performed with patients or patient material, and hence no approval or consent was required. Human cell lines have been purchased from ATCC. Mouse cell lines have been derived under the Animal Experiment Permissions 1887, 1907, and 1908 by the Swiss Authorities (Kantonale Veterinäramt Basel-Stadt).

\section{Consent for publication}

Not applicable.

\section{Competing interests}

The authors declare that they have no competing interests.

\section{Publisher's Note}

Springer Nature remains neutral with regard to jurisdictional claims in published maps and institutional affiliations.

\section{Author details}

'Department of Biomedicine, University of Basel, Mattenstrasse 28, 4058 Basel, Switzerland. 2Present address: Institute of Pathology, University Hospital of Basel, Basel, Switzerland. ${ }^{3}$ Present address: Integra Biosciences AG, Zizers, Switzerland. ${ }^{4}$ Present address: Roche Pharma, Basel, Switzerland.

\section{Received: 23 March 2018 Accepted: 20 August 2018}

Published online: 01 October 2018

\section{References}

1. Yang J, Weinberg RA. Epithelial-mesenchymal transition: at the crossroads of development and tumor metastasis. Dev Cell. 2008;14(6):818-29.

2. Kalluri R. EMT: when epithelial cells decide to become mesenchymal-like cells. J Clin Invest. 2009;119(6):1417-9.

3. Thiery JP, Acloque H, Huang RY, Nieto MA. Epithelial-mesenchymal transitions in development and disease. Cell. 2009;139(5):871-90.

4. Chaffer CL, San Juan BP, Lim E, Weinberg RA. EMT, cell plasticity and metastasis. Cancer Metastasis Rev. 2016;35(4):645-54.

5. Nieto MA. Epithelial plasticity: a common theme in embryonic and cancer cells. Science. 2013;342(6159):1234850.

6. Bloushtain-Qimron N, Yao J, Snyder EL, Shipitsin M, Campbell LL, Mani SA, Hu M, Chen H, Ustyansky V, Antosiewicz JE, et al. Cell type-specific DNA methylation patterns in the human breast. Proc Natl Acad Sci U S A. 2008; 105(37):14076-81.

7. Shipitsin M, Campbell L, Argani P, Weremowicz S, Bloushtain-Qimron N, Yao J, Nikolskaya T, Serebryiskaya T, Beroukhim R, Hu M, et al. Molecular definition of breast tumor heterogeneity. Cancer Cell. 2007;11(3):259-73.

8. Graff JR, Gabrielson E, Fujii H, Baylin SB, Herman JG. Methylation patterns of the E-cadherin 5' CpG island are unstable and reflect the dynamic, heterogeneous loss of E-cadherin expression during metastatic progression. J Biol Chem. 2000;275(4):2727-32.

9. Shipitsin M, Polyak K. The cancer stem cell hypothesis: in search of definitions, markers, and relevance. Lab Invest. 2008;88(5):459-63.

10. Brabletz T, Hlubek F, Spaderna S, Schmalhofer O, Hiendlmeyer E, Jung A, Kirchner T. Invasion and metastasis in colorectal cancer: epithelialmesenchymal transition, mesenchymal-epithelial transition, stem cells and beta-catenin. Cells Tissues Organs. 2005;179(1-2):56-65.

11. Chaffer C, Thompson E, Williams E. Mesenchymal to epithelial transition in development and disease. Cells Tissues Organs. 2007;185(1-3):7-19.

12. Ocana OH, Corcoles R, Fabra A, Moreno-Bueno G, Acloque H, Vega S, Barrallo-Gimeno A, Cano A, Nieto MA. Metastatic colonization requires the repression of the epithelial-mesenchymal transition inducer Prrx1. Cancer Cell. 2012;22(6):709-24.

13. Tsai JH, Donaher JL, Murphy DA, Chau S, Yang J. Spatiotemporal regulation of epithelial-mesenchymal transition is essential for squamous cell carcinoma metastasis. Cancer Cell. 2012;22(6):725-36.

14. Fischer KR, Durrans A, Lee S, Sheng J, Li F, Wong ST, Choi H, El Rayes T, Ryu $\mathrm{S}$, Troeger J, et al. Epithelial-to-mesenchymal transition is not required for lung metastasis but contributes to chemoresistance. Nature. 2015;527(7579): 472-6.

15. Zheng X, Carstens JL, Kim J, Scheible M, Kaye J, Sugimoto H, Wu CC, LeBleu VS, Kalluri R. Epithelial-to-mesenchymal transition is dispensable for metastasis but induces chemoresistance in pancreatic cancer. Nature. 2015; 527(7579):525-30
16. Aiello NM, Brabletz T, Kang Y, Nieto MA, Weinberg RA, Stanger BZ. Upholding a role for EMT in pancreatic cancer metastasis. Nature. 2017; 547(7661):E7-8.

17. Ye X, Brabletz T, Kang Y, Longmore GD, Nieto MA, Stanger BZ, Yang J, Weinberg RA. Upholding a role for EMT in breast cancer metastasis. Nature. 2017:547(7661):E1-3.

18. Krebs AM, Mitschke J, Lasierra Losada M, Schmalhofer O, Boerries M, Busch H, Boettcher M, Mougiakakos D, Reichardt W, Bronsert P, et al. The EMTactivator Zeb1 is a key factor for cell plasticity and promotes metastasis in pancreatic cancer. Nat Cell Biol. 2017;19(5):518-29.

19. Polyak K, Weinberg RA. Transitions between epithelial and mesenchymal states: acquisition of malignant and stem cell traits. Nat Rev Cancer. 2009; 9(4):265-73.

20. Yilmaz M, Maass D, Tiwari N, Waldmeier L, Schmidt P, Lehembre F, Christofori G. Transcription factor Dlx2 protects from TGFbeta-induced cellcycle arrest and apoptosis. EMBO J. 2011;30(21):4489-99.

21. Tiwari N, Meyer-Schaller N, Arnold P, Antoniadis H, Pachkov M, van Nimwegen E, Christofori G. Klf4 is a transcriptional regulator of genes critical for EMT, including Jnk1 (Mapk8). PLoS One. 2013;8(2):e57329.

22. Tiwari $N$, Tiwari VK, Waldmeier $L$, Balwierz PJ, Arnold P, Pachkov M, MeyerSchaller N, Schubeler D, van Nimwegen E, Christofori G. Sox4 is a master regulator of epithelial-mesenchymal transition by controlling Ezh2 expression and epigenetic reprogramming. Cancer Cell. 2013;23(6):768-83.

23. Lehembre F, Yilmaz M, Wicki A, Schomber T, Strittmatter K, Ziegler D, Kren A, Went P, Derksen PW, Berns A, et al. NCAM-induced focal adhesion assembly: a functional switch upon loss of E-cadherin. EMBO J. 2008;27(19): 2603-15.

24. Kaufmann $E$, Knochel W. Five years on the wings of fork head. Mech Dev. 1996;57(1):3-20.

25. Kaestner $\mathrm{KH}$, Knochel W, Martinez DE. Unified nomenclature for the winged helix/forkhead transcription factors. Genes Dev. 2000;14(2):142-6.

26. Wotton KR, Shimeld SM. Analysis of lamprey clustered fox genes: insight into fox gene evolution and expression in vertebrates. Gene. 2011;489(1):30-40.

27. Fuxe J, Vincent T, Garcia de Herreros A. Transcriptional crosstalk between TGF-beta and stem cell pathways in tumor cell invasion: role of EMT promoting Smad complexes. Cell Cycle. 2010;9(12):2363-74.

28. Mani SA, Yang J, Brooks M, Schwaninger G, Zhou A, Miura N, Kutok JL, Hartwell K, Richardson AL, Weinberg RA. Mesenchyme forkhead 1 (FOXC2) plays a key role in metastasis and is associated with aggressive basal-like breast cancers. Proc Natl Acad Sci U S A. 2007;104(24):10069-74.

29. Taube JH, Herschkowitz Jl, Komurov K, Zhou AY, Gupta S, Yang J, Hartwell K, Onder T, Gupta PB, Evans KW, et al. Core epithelial-to-mesenchymal transition interactome gene-expression signature is associated with claudinlow and metaplastic breast cancer subtypes. Proc Natl Acad Sci U S A. 2010; 107(35):15449-54

30. Bao B, Wang Z, Ali S, Kong D, Banerjee S, Ahmad A, Li Y, Azmi AS, Miele L, Sarkar FH. Over-expression of FoxM1 leads to epithelial-mesenchymal transition and cancer stem cell phenotype in pancreatic cancer cells. J Cell Biochem. 2011;112(9):2296-306.

31. Aitola M, Carlsson P, Mahlapuu M, Enerback S, Pelto-Huikko M. Forkhead transcription factor FoxF2 is expressed in mesodermal tissues involved in epithelio-mesenchymal interactions. Dev Dyn. 2000;218(1):136-49.

32. Blixt A, Mahlapuu M, Bjursell C, Darnfors $C$, Johannesson T, Enerback $S$, Carlsson P. The two-exon gene of the human forkhead transcription factor FREAC-2 (FKHL6) is located at 6p25.3. Genomics. 1998;53(3):387-90.

33. Hellqvist M, Mahlapuu M, Blixt A, Enerback S, Carlsson P. The human forkhead protein FREAC-2 contains two functionally redundant activation domains and interacts with TBP and TFIIB. J Biol Chem. 1998;273(36):23335-43.

34. Lan $Y$, Jiang R. Sonic hedgehog signaling regulates reciprocal epithelialmesenchymal interactions controlling palatal outgrowth. Development. 2009:136(8):1387-96.

35. Katoh Y, Katoh M. Hedgehog signaling, epithelial-to-mesenchymal transition and miRNA (review). Int J Mol Med. 2008;22(3):271-5.

36. Yu W, Ruest LB, Svoboda KK. Regulation of epithelial-mesenchymal transition in palatal fusion. Exp Biol Med (Maywood). 2009;234(5):483-91.

37. Wilkie AO, Morriss-Kay GM. Genetics of craniofacial development and malformation. Nat Rev Genet. 2001;2(6):458-68.

38. Wang T, Tamakoshi T, Uezato T, Shu F, Kanzaki-Kato N, Fu Y, Koseki H, Yoshida N, Sugiyama T, Miura N. Forkhead transcription factor Foxf2 (LUN)deficient mice exhibit abnormal development of secondary palate. Dev Biol. 2003;259(1):83-94 
39. Ormestad M, Astorga J, Landgren $H$, Wang T, Johansson BR, Miura N, Carlsson P. Foxf1 and Foxf2 control murine gut development by limiting mesenchymal Wnt signaling and promoting extracellular matrix production. Development. 2006;133(5):833-43.

40. Shi W, Gerster K, Alajez NM, Tsang J, Waldron L, Pintilie M, Hui AB, Sykes J, P'ng C, Miller N, et al. MicroRNA-301 mediates proliferation and invasion in human breast cancer. Cancer Res. 2011;71(8):2926-37.

41. Lo HW, Hsu SC, Xia W, Cao X, Shih JY, Wei Y, Abbruzzese JL, Hortobagyi GN, Hung MC. Epidermal growth factor receptor cooperates with signal transducer and activator of transcription 3 to induce epithelialmesenchymal transition in cancer cells via up-regulation of TWIST gene expression. Cancer Res. 2007;67(19):9066-76.

42. Nik AM, Reyahi A, Ponten F, Carlsson P. Foxf2 in intestinal fibroblasts reduces numbers of $\operatorname{Lgr5}(+)$ stem cells and adenoma formation by inhibiting Wnt signaling. Gastroenterology. 2013;144(5):1001-11.

43. Kong PZ, Yang F, Li L, Li XQ, Feng YM. Decreased FOXF2 mRNA expression indicates early-onset metastasis and poor prognosis for breast cancer patients with histological grade II tumor. PLoS One. 2013;8(4):e61591.

44. Cai J, Tian AX, Wang QS, Kong PZ, Du X, Li XQ, Feng YM. FOXF2 suppresses the FOXC2-mediated epithelial-mesenchymal transition and multidrug resistance of basal-like breast cancer. Cancer Lett. 2015;367(2):129-37.

45. Wang QS, Kong PZ, Li XQ, Yang F, Feng YM. FOXF2 deficiency promotes epithelial-mesenchymal transition and metastasis of basal-like breast cancer. Breast Cancer Res. 2015;17:30.

46. Lo PK. The controversial role of forkhead box F2 (FOXF2) transcription factor in breast cancer. PRAS Open. 2017;1.

47. Maeda M, Johnson KR, Wheelock MJ. Cadherin switching: essential for behavioral but not morphological changes during an epithelium-tomesenchyme transition. J Cell Sci. 2005;118(Pt 5):873-87.

48. Deckers M, van Dinther M, Buijs J, Que I, Lowik C, van der Pluijm G, ten Dijke $P$. The tumor suppressor Smad4 is required for transforming growth factor beta-induced epithelial to mesenchymal transition and bone metastasis of breast cancer cells. Cancer Res. 2006;66(4):2202-9.

49. Waldmeier L, Meyer-Schaller N, Diepenbruck M, Christofori G. Py2T murine breast cancer cells, a versatile model of TGFbeta-induced EMT in vitro and in vivo. PLoS One. 2012;7(11):e48651.

50. Hellqvist M, Mahlapuu M, Samuelsson L, Enerback S, Carlsson P. Differential activation of lung-specific genes by two forkhead proteins, FREAC-1 and FREAC-2. J Biol Chem. 1996;271(8):4482-90.

51. Weber M, Hellmann I, Stadler MB, Ramos L, Paabo S, Rebhan M, Schubeler D. Distribution, silencing potential and evolutionary impact of promoter DNA methylation in the human genome. Nat Genet. 2007;39(4):457-66.

52. Muller WJ, Sinn E, Pattengale PK, Wallace R, Leder P. Single-step induction of mammary adenocarcinoma in transgenic mice bearing the activated cneu oncogene. Cell. 1988;54(1):105-15.

53. Derksen PW, Liu X, Saridin F, van der Gulden H, Zevenhoven J, Evers B, van Beijnum JR, Griffioen AW, Vink J, Krimpenfort P, et al. Somatic inactivation of E-cadherin and p53 in mice leads to metastatic lobular mammary carcinoma through induction of anoikis resistance and angiogenesis. Cancer Cell. 2006;10(5):437-49.

54. Piek E, Moustakas A, Kurisaki A, Heldin CH, ten Dijke P. TGF-(beta) type I receptor/ALK-5 and Smad proteins mediate epithelial to mesenchymal transdifferentiation in NMuMG breast epithelial cells. J Cell Sci. 1999;112(Pt 24):4557-68

55. Brabletz S, Brabletz T. The ZEB/miR-200 feedback loop-a motor of cellular plasticity in development and cancer? EMBO Rep. 2010;11(9):670-7.

56. Caramel J, Ligier M, Puisieux A. Pleiotropic roles for ZEB1 in cancer. Cancer Res. 2018;78(1):30-5.

57. Gheldof A, Hulpiau P, van Roy F, De Craene B, Berx G. Evolutionary functional analysis and molecular regulation of the ZEB transcription factors. Cell Mol Life Sci. 2012;69(15):2527-41.

58. Kondo M, Cubillo E, Tobiume K, Shirakihara T, Fukuda N, Suzuki H, Shimizu K, Takehara K, Cano A, Saitoh M, et al. A role for Id in the regulation of TGFbeta-induced epithelial-mesenchymal transdifferentiation. Cell Death Differ. 2004;11(10):1092-101.

59. Derynck R, Akhurst RJ, Balmain A. TGF-beta signaling in tumor suppression and cancer progression. Nat Genet. 2001;29(2):117-29.

60. Fabregat I, Herrera B, Fernandez M, Alvarez AM, Sanchez A, Roncero C, Ventura JJ, Valverde AM, Benito M. Epidermal growth factor impairs the cytochrome C/caspase-3 apoptotic pathway induced by transforming growth factor beta in rat fetal hepatocytes via a phosphoinositide 3-kinasedependent pathway. Hepatology. 2000;32(3):528-35.

61. Murillo MM, del Castillo G, Sanchez A, Fernandez M, Fabregat I. Involvement of EGF receptor and c-Src in the survival signals induced by TGF-beta1 in hepatocytes. Oncogene. 2005;24(28):4580-7.

62. Minn A, Gupta G, Siegel P, Bos P, Shu W, Giri D, Viale A, Olshen A, Gerald W, Massague J. Genes that mediate breast cancer metastasis to lung. Nature. 2005;436(7050):518-24.

63. van 't Veer $L J$, Dai $H$, van de Vijver MJ, He YD, Hart AA, Mao M, Peterse $H L$, van der Kooy K, Marton MJ, Witteveen AT, et al. Gene expression profiling predicts clinical outcome of breast cancer. Nature. 2002;415(6871):530-6.

64. Curtis C, Shah SP, Chin SF, Turashvili G, Rueda OM, Dunning MJ, Speed D, Lynch AG, Samarajiwa S, Yuan Y, et al. The genomic and transcriptomic architecture of 2,000 breast tumours reveals novel subgroups. Nature. 2012; 486(7403):346-52.

65. Dvinge H, Git A, Graf S, Salmon-Divon M, Curtis C, Sottoriva A, Zhao Y, Hirst M, Armisen J, Miska EA, et al. The shaping and functional consequences of the microRNA landscape in breast cancer. Nature. 2013;497(7449):378-82.

66. Thiery JP, Sleeman JP. Complex networks orchestrate epithelialmesenchymal transitions. Nat Rev Mol Cell Biol. 2006;7(2):131-42.

67. Birchmeier W, Behrens J. Cadherin expression in carcinomas: role in the formation of cell junctions and the prevention of invasiveness. Biochim Biophys Acta. 1994;1198(1):11-26.

68. Christofori G. New signals from the invasive front. Nature. 2006;441(7092):444-50.

69. Kalluri R, Weinberg R. The basics of epithelial-mesenchymal transition. J Clin Invest 2009:119(6):1420-8.

70. Peinado H, Olmeda D, Cano A. Snail, Zeb and bHLH factors in tumour progression: an alliance against the epithelial phenotype? Nat Rev Cancer. 2007;7(6):415-28.

71. Perk J, lavarone A, Benezra R. Id family of helix-loop-helix proteins in cancer. Nat Rev Cancer. 2005;5(8):603-14.

72. Park SM, Gaur AB, Lengyel E, Peter ME. The miR-200 family determines the epithelial phenotype of cancer cells by targeting the E-cadherin repressors ZEB1 and ZEB2. Genes Dev. 2008;22(7):894-907.

73. Gregory PA, Bert AG, Paterson EL, Barry SC, Tsykin A, Farshid G, Vadas MA, Khew-Goodall Y, Goodall GJ. The miR-200 family and miR-205 regulate epithelial to mesenchymal transition by targeting ZEB1 and SIP1. Nat Cell Biol. 2008;10(5):593-601.

74. Burk U, Schubert J, Wellner U, Schmalhofer O, Vincan E, Spaderna S, Brabletz T. A reciprocal repression between ZEB1 and members of the miR-200 family promotes EMT and invasion in cancer cells. EMBO Rep. 2008;9(6):582-9.

75. Kundu ST, Byers LA, Peng DH, Roybal JD, Diao L, Wang J, Tong P, Creighton CJ, Gibbons DL. The miR-200 family and the miR-183 96 182 cluster target Foxf2 to inhibit invasion and metastasis in lung cancers. Oncogene. 2016; 35(2):173-86.

76. Willis SN, Chen L, Dewson G, Wei A, Naik E, Fletcher Jl, Adams JM, Huang DC. Proapoptotic Bak is sequestered by $\mathrm{MCl}-1$ and $\mathrm{BCl}-\mathrm{xL}$, but not $\mathrm{BCl}-2$, until displaced by BH3-only proteins. Genes Dev. 2005;19(11):1294-305.

77. Kim JY, Ahn HJ, Ryu JH, Suk K, Park JH. BH3-only protein Noxa is a mediator of hypoxic cell death induced by hypoxia-inducible factor 1alpha. J Exp Med. 2004;199(1):113-24.

78. Ploner C, Kofler R, Villunger A. Noxa: at the tip of the balance between life and death. Oncogene. 2009;27:S84-92.

79. Dairkee SH, Ljung BM, Smith H, Hackett A. Immunolocalization of a human basal epithelium specific keratin in benign and malignant breast disease. Breast Cancer Res Treat. 1987;10(1):11-20. 